\title{
On the recent star formation history of the Milky Way disk
}

\author{
R. de la Fuente Marcos \\ Suffolk University Madrid Campus, C/ Viña, 3. E-28003 Madrid, Spain \\ raul@galaxy.suffolk.es \\ C. de la Fuente Marcos \\ Universidad Complutense de Madrid, E-28040 Madrid, Spain \\ nbplanet@fis.ucm.es
}

\begin{abstract}
We have derived the star formation history of the Milky Way disk over the last 2 Gyr with a time resolution of 0.05 Gyr from the age distribution diagram of a large sample of open clusters comprising more than 580 objects. By interpreting the age distribution diagram using numerical results from an extensive library of $N$-body calculations carried out during the last ten years, we reconstruct the recent star formation history of the Milky Way disk. Under the assumption that the disk has never been polluted by any extragalactic stellar populations, our analysis suggests that superimposed on a relatively small level of constant star formation activity mainly in small- $N$ star clusters, the star formation rate has experienced at least 5 episodes of enhanced star formation lasting about 0.2 Gyr with production of larger clusters. This cyclic behavior shows a period of $0.4 \pm 0.1$ Gyr and could be the result of density waves and/or interactions with satellite galaxies. On the other hand, the star formation rate history from a volume-limited sample of open clusters in the solar neighbourhood appears to be consistent with the overall star formation history obtained from the entire sample. Pure continuous star formation both in the solar neighbourhood and the entire Galactic disk is strongly ruled out. Our results also indicate that, in the Milky Way disk, about $90 \%$ of open clusters are born with $N \leq 150$ and the slope in the power-law frequency distribution of their masses is about -2.7 when quiescent star formation takes place. If the above results are re-interpreted taking into consideration accretion events onto the Milky Way, it is found that a fraction of the unusually high number of open clusters with ages older than 0.6 Gyr may have been formed in disrupted satellites. Problems arising from the selection effects and the age errors in the open cluster sample used are discussed in detail.
\end{abstract}

Key words: Galaxy: disk - Galaxy: evolution - Galaxy: open clusters and associations: general - solar neighbourhood - star formation

Preprint submitted to Elsevier Science 29 October 2018 


\section{Introduction}

Throughout the Milky Way's history, a non-negligible fraction of star formation has apparently occurred in starburst-like events (see Majewski, 1993 or Freeman \& Bland-Hawthorn, 2002, for a review). On the other hand, observations suggest that present-day star formation in the disk of our Galaxy takes place in stellar groupings rather than in isolation. These stellar aggregates form from molecular clouds, and in the disk of the Milky Way they appear in two types: bound and unbound. Unbound, short lived stellar groupings are called associations; bound, long lived stellar groups are known as open clusters. Open clusters can also be formed out of the remains of rich stellar associations (Kroupa et al., 2001). As most of the field stars appear to have been formed in the so-called clustered mode (i.e., in clusters or associations), not in the dispersed mode (i.e., in isolation), it is a natural choice to consider these stellar clumpings as the de facto units of star formation in the disk of our Galaxy (Clarke et al., 2000). The idea of open clusters being fundamental units of star formation in the Galactic disk is, however, controversial (Meyer et al., 2000). It has been argued that bound open clusters cannot contribute significantly to the field star population of the Galactic disk because they are rare and long lived (e.g. Roberts, 1957). In contrast, most young embedded clusters are thought to evolve into unbound stellar associations, which comprise the majority of stars that populate the Milky Way disk (Lada \& Lada, 1991). Nevertheless, if star clusters are the elementary units of star formation they can, in principle, be used to derive the star formation history, recent and old. Unfortunately, stellar associations evolve and dissolve in a time-scale of $\sim 50$ Myr (Brown, 2002), therefore they cannot be used to study the star formation history of the Galactic disk. On the other hand, open clusters are comparatively long lived objects that may serve as excellent probes into the structure and evolution of the Galactic disk.

The problem of deriving the star formation history of the Milky Way has been considered by a number of authors using different techniques (Twarog, 1980; Scalo, 1987; Barry, 1988; Gomez et al., 1990; Marsakov et al., 1990; Noh \& Scalo, 1990; Meusinger, 1991; Soderblom et al., 1991; Micela et al., 1993; Díaz-Pinto et al., 1994; Rocha-Pinto \& Maciel, 1997; Chereul et al., 1998; Isern et al., 1999; Lachaume et al., 1999; Rocha-Pinto et al., 2000a; Hernandez et al., 2000a; Bertelli \& Nasi, 2001; Gizis et al., 2002; Just, 2002, 2003). The majority of these studies suggest that the disk of the Milky Way Galaxy has not experienced a smooth and constant star formation history but a bursty one with several episodes of enhanced star formation. Although most of these papers are restricted to the study of the star formation history in the 
solar neighbourhood, the star sample birth sites are in fact distributed over a larger range of distances because of orbital diffusion, and so they can provide an estimate of the global star formation rate and their conclusions can be extrapolated to the entire Milky Way disk.

In this paper we revisit the topic of the recent star formation history of the Milky Way disk by using data from the Open Cluster Database (Mermilliod, 2003), the New Catalogue of Optically Visible Open Clusters and Candidates (Dias et al., 2003a, b) as well as Hipparcos data (ESA, 1997) to construct the open cluster age distribution. The age distribution diagram is then interpreted using numerical results from an extensive library of $N$-body calculations carried out during the last ten years. This method permits the reconstruction of the star formation history of the Milky Way disk over the last 2 Gyr with a time resolution of 0.05 Gyr using very few a priori assumptions. Our main objective is to understand the recent star formation history of the entire Galactic disk although only the solar circle $( \pm 1.75 \mathrm{kpc})$ can be studied due to the incompleteness of the available open cluster sample (see Section 2).

This paper is organized as follows: in Section 2, we present the properties of the Galactic open cluster sample. The raw histogram of open cluster number vs. age is also included in this Section as well as a discussion of the potential limitations of our approach. In Section 3, we summarize some relevant results on the evolution and dissolution of realistic $N$-body star cluster models. This Section also includes a comparison between published open cluster ages and number of stars with the results of the numerical models. In Section 4, we present our method as well as results for the Galactic disk. A closer view to the last 0.2 Gyr and the derived open cluster initial mass function are considered in Section 5. The star formation history is presented and discussed in Section 6 ; corrections are also discussed here. A detailed comparison with results from other authors is carried out in Section 7. An alternative interpretation of our results in the context of dynamical merger histories is included in Section 8. Open questions and conclusions are summarized in Section 9.

\section{An open cluster sample}

For many years, the Lyngå catalogue $(1985,1987)$ has been the classical reference source for open cluster data. An exhaustive analysis of the properties of the Milky Way open cluster system using this catalogue was carried out by Janes et al. (1988). Unfortunately, the Lyngå catalogue is not the most updated source of data in this field. The Open Cluster Database (WEBDA) created and maintained by J.-C. Mermilliod (1988, 1992a, 1992b, 1993, 1995, 1996, 2003) includes all the data already covered in Lyngå's catalogue and many more. The latest update of the Open Cluster Database (October 2003) 
includes 1731 open clusters with ages for 616 objects (36\%). In this database we have found 581 clusters with age $\leq 2$ Gyr. Slightly less complete is the New Catalogue of Optically Visible Open Clusters and Candidates (NCOVOCC). The first version of this catalogue was published in Dias et al. (2002). The October 2003 version (Dias et al., 2003b), includes 1637 objects, 603 (37\%) with published ages. This new catalogue updates the previous catalogues of Lyngå (1987) and of Mermilliod (1995) included in the WEBDA database. There are however non-negligible differences between the two databases as we can see from Figs. 1 and 2. NCOVOCC includes 569 clusters of age $\leq 2$ Gyr. On the other hand, Salaris et al. (2004) have recently re-determined ages for a sample of 71 of the oldest open clusters (all of them older than 0.67 Gyr) using a morphological age indicator, metallicities, and Hipparcos parallaxes. In general, the new ages are older than those from WEBDA and NCOVOCC. In order to study the impact of different age determination techniques on our results we have modified the NCOVOCC data with the new age determinations from Salaris et al. (2004). The new subsample consists of 559 open clusters younger than 2 Gyr. The age-distance diagram for this new sample appears in Fig. 3.

In this paper we use the three subsamples presented above to construct an age distribution diagram. As explained below, we choose 2 Gyr in order to obtain statistically relevant results. Our samples contain objects from dense, very young clusters to old and almost dissolved moving groups. The age distribution diagram for the three open cluster samples considered is shown in Fig. 4. Although a detailed analysis of the different features present in this histogram is postponed to Section 4, the figure suggests that several epochs of enhanced star formation may have taken place during the last 2 Gyr.

The age-distance diagrams for the three samples appear in Figs. 1, 2, and 3. These figures indicate that the open cluster data used in this paper provide an incomplete sample; unfortunately, it is difficult to estimate the degree of incompleteness of this sample due to the irregular distribution of Galactic open clusters. The sample appears to be rather incomplete for open clusters more distant than $\sim 3.5 \mathrm{kpc}$, therefore our conclusions can only be rigorously applied to the solar circle: an annulus of Galactocentric distance $R_{\odot} \pm 1.75 \mathrm{kpc}$, where $R_{\odot}$ is the solar Galactocentric distance ( $~ 8.5 \mathrm{kpc}$, e.g., Zombeck, 1990). On the other hand, the Hipparcos Catalogue contains numerous selection biases which are difficult to characterize. It is, however, reasonable to assume that the sample is fairly complete (166 clusters) for objects in the solar neighbourhood (distances to the Sun of $1 \mathrm{kpc}$ or less) although selection effects are likely to be very important for clusters located in the outskirts of the Galactic disk as well as in the region behind the Galactic center and for small clusters with Galactocentric distance $<8.5 \mathrm{kpc}$ as they are projected against the dense stellar fields of the southern Milky Way. On the other hand, intermediate age and old open cluster samples are incomplete as clusters with higher $|z|$ are 


\section{Mermilliod (2003)}

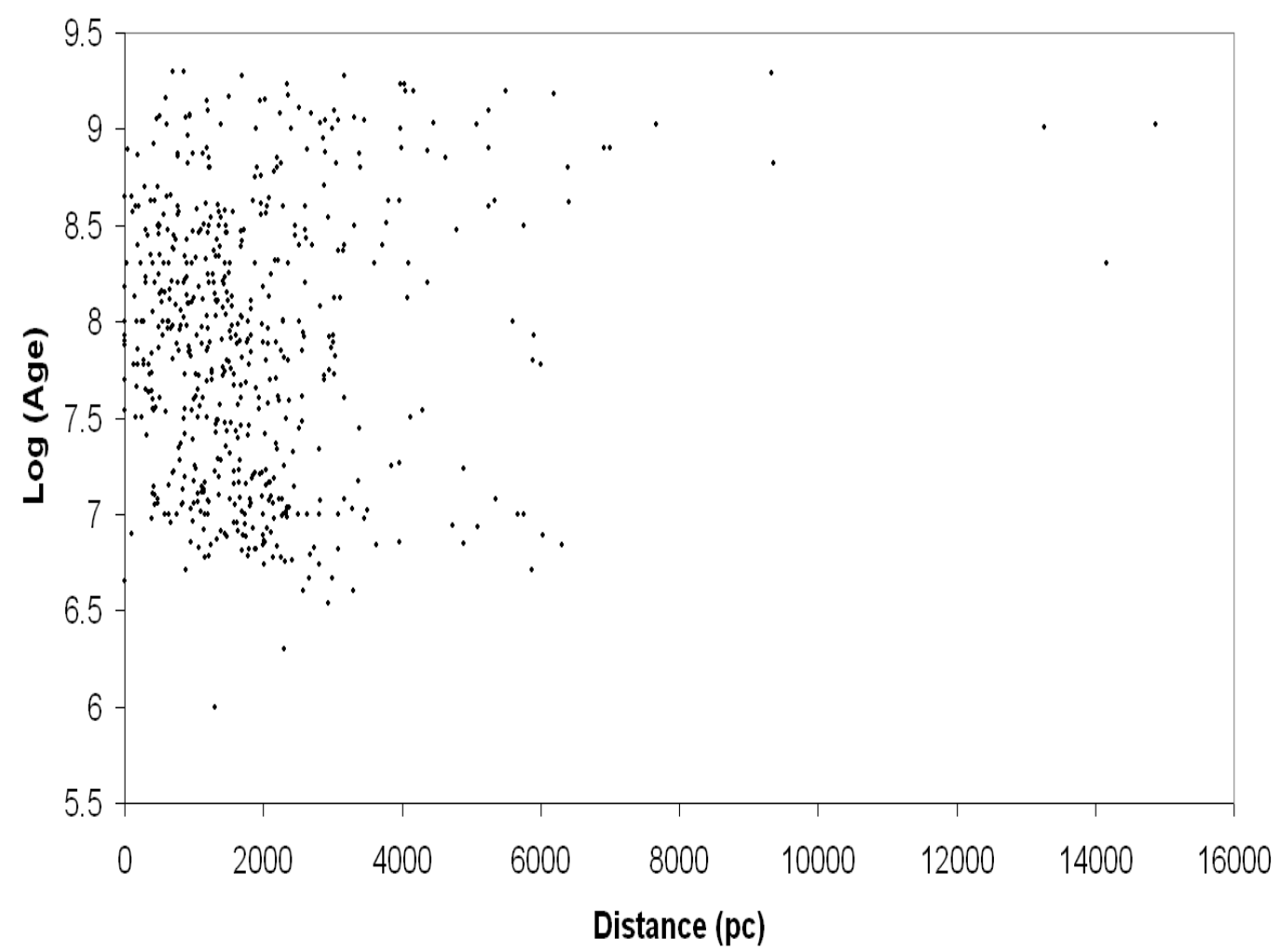

Fig. 1. Age-distance diagram of the open cluster sample from the latest update (October 2003) of WEBDA (Mermilliod, 2003). Only clusters with age $\leq 2$ Gyr (581 objects) are plotted. The sample appears to be rather incomplete for open clusters more distant than $3.5 \mathrm{kpc}$.

easier to detect than clusters with lower $|z|$. In this paper, we are interested in studying only the recent star formation history of the Milky Way ( $\leq 2 \mathrm{Gyr}$ ), therefore any effects arising from lack of completeness of the sample are likely to be less important than those from age errors. Besides, open cluster samples are biased toward objects where the total luminosity is dominated by ultraluminous $\mathrm{O}$ and $\mathrm{B}$ stars (young clusters) or red (or blue) giants (old clusters) as these systems are easier to identify. However, if $\mathrm{O}$ and $\mathrm{B}$ stars were the only stars the cluster would not be stable since the massive stars are destined to become supernovae, returning their mass to the interstellar medium. Hence, the existence of low mass stars is critical for the survival of open clusters. Old, dynamically depleted open clusters are very difficult to identify although they may exist in large numbers (de la Fuente Marcos, 1998). Faint, population depleted open clusters are very difficult to identify against the field stars. Therefore we are working with an incomplete, volume-limited sample that is also flux-limited. 


\section{Dias et al. (2003b)}

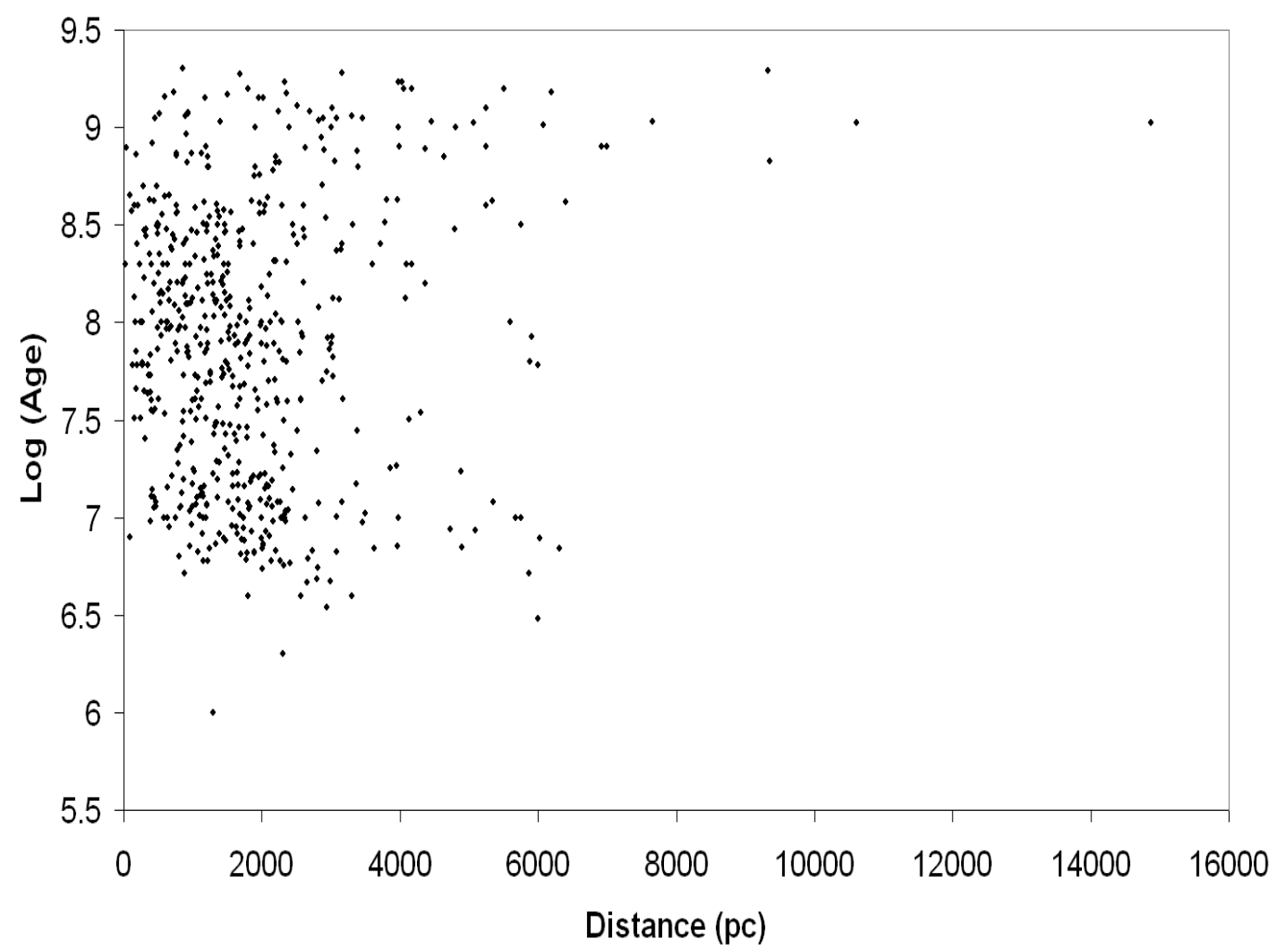

Fig. 2. Age-distance diagram of the open cluster sample from the latest version of the New Catalogue of Optically Visible Open Clusters and Candidates (Dias et al., 2003b) for clusters of age $\leq 2$ Gyr (569 objects). There is a strong selection effect as the number of open clusters more distant than $3.5 \mathrm{kpc}$ is small in our sample.

On the other hand, and as for any other physical parameter, open cluster age determinations are affected by errors. In a large sample, these errors are very likely to be non-homogeneous as different methods have been used by different authors to calculate the ages. The disparity in open cluster ages (when considering different authors, see WEBDA for multiple examples) partly reflects the critical dependence of cluster isochrone fitting on the adopted reddening (often large for open clusters), even small reddening errors can create significant errors in the derived age and metallicity. However, determining effective temperatures and metallicities for cluster turn off stars directly through echelle spectroscopy is free from systematic errors and subject only to uncertainties in the model atmospheres. Some ages for clusters in the samples considered have been determined using the first technique, but some others have been found using the second one or even other indirect methods. It is relatively difficult, specially in papers older than about 5 years, to find published estimations of the errors associated with open cluster age determinations. In order to evaluate the impact of age errors in our analysis we have compiled errors for 52 
Dias et al. (2003b) modified with Salaris et al. (2004)

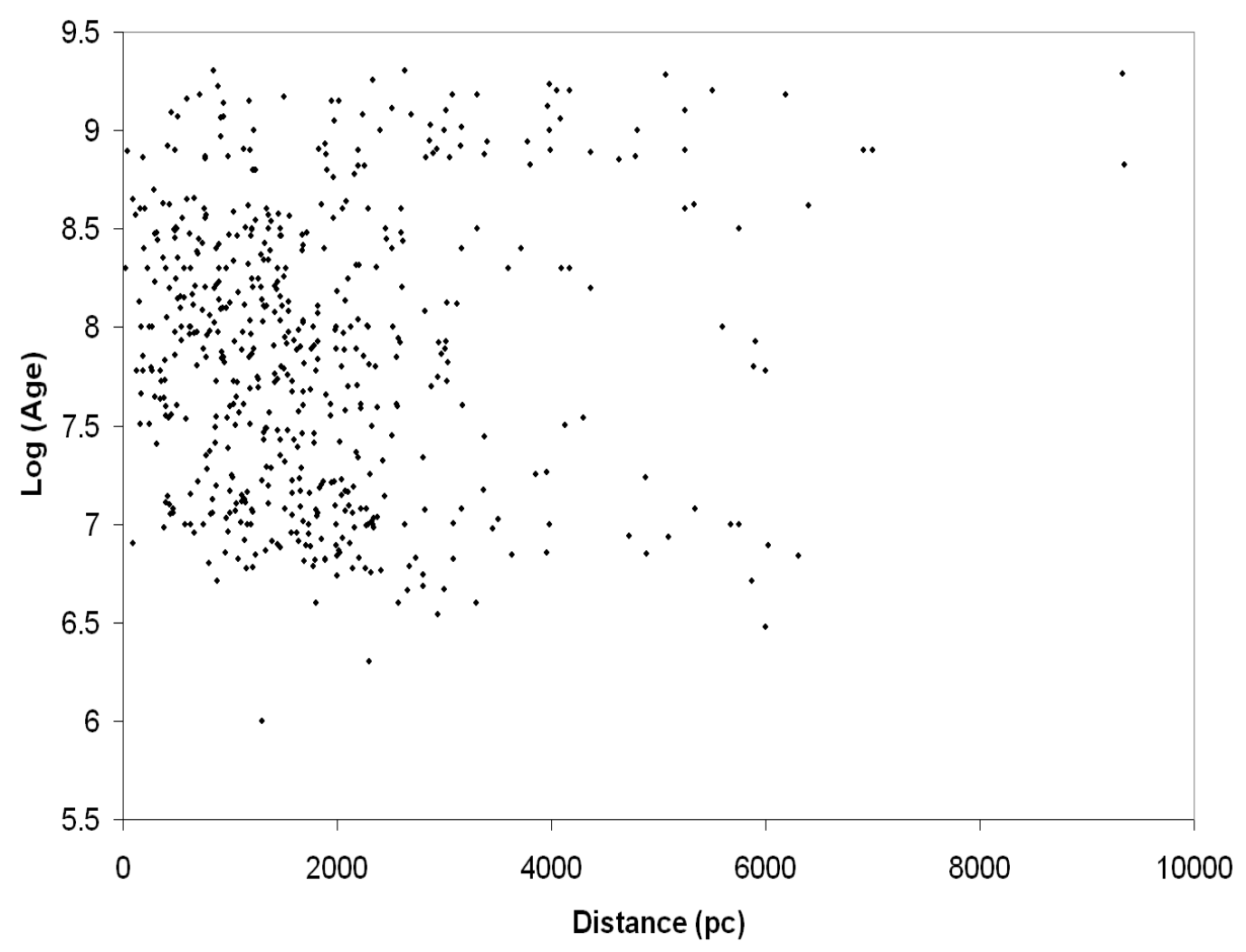

Fig. 3. Age-distance diagram of the open cluster sample (559 objects) in Fig. 2 modified with new age determinations by Salaris et al. (2004). This sample includes less objects and covers a different distance range because some of the new ages are older ( $>2$ Gyr) than those in the two previous samples.

objects within the studied age range from published sources: NGC 6134 and NGC 3680 (Bruntt et al., 1999); NGC 2141 (Carraro et al., 2001); NGC 2158 (Grocholski \& Sarajedini, 2002); NGC 2112 (Carraro et al., 2002); Berkeley 104, Berkeley 60, King 15, NGC 381, Berkeley 64, King 6, NGC 1348, Berkeley 23, NGC 2259, NGC 2304 (Ann et al., 2002); NGC 1663 (Baume et al., 2003); NGC 3990 (Prisinzano et al., 2004); Pismis 19 (Carraro \& Munari, 2004); IC 166, NGC 752, King 5, NGC 1254, NGC 1278, NGC 1817, NGC 2158, NGC 2194, NGC 2192, NGC 2236, NGC 2266, Berkeley 30, NGC 2324, NGC 2354, NGC 2355, NGC 2360, Haffner 6, Melotte 71, Pismis 2, NGC 2660, NGC 2849, NGC 3680, NGC 4815, NGC 5822, IC 4651, IC 4756, Berkeley 42, NGC 6802, NGC 6827, NGC 7044, NGC 2477, NGC 7789, NGC 2204, Hyades, and Praesepe (Salaris et al., 2004). Fig. 5 shows the uncertainty as a function of the age for this open cluster sample. The average age error is about $22 \%$ and the plot suggests that younger ages are affected by larger errors (for a recent discussion on the problems associated with age determinations in young open clusters see, e.g., Piskunov et al., 2004). If this error sample can be considered 


\section{Open Cluster Age Distribution}

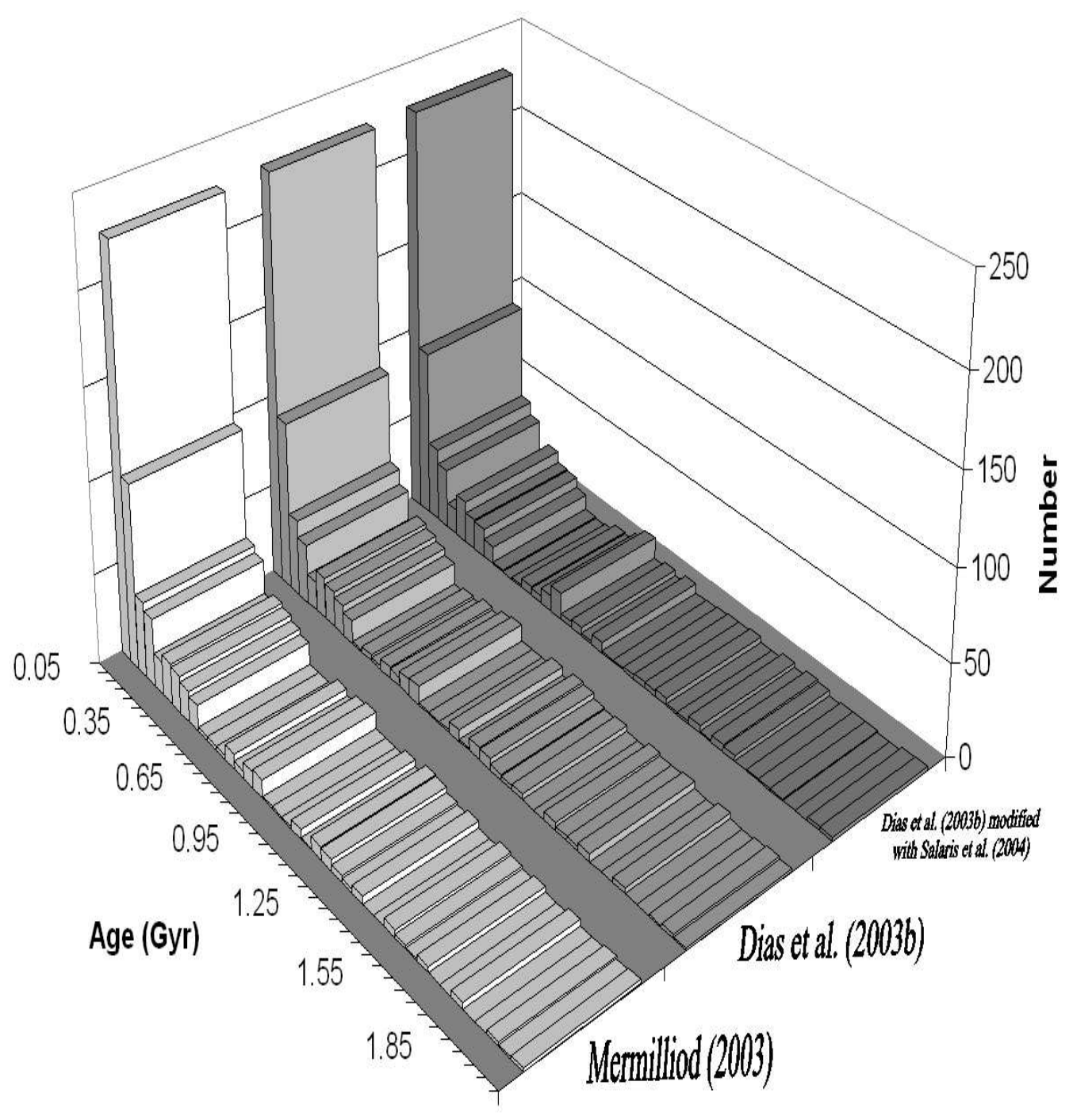

Fig. 4. Open cluster age distribution from the last update (October 2003) of WEBDA (Mermilliod, 2003), white, the latest version (October 2003) of the New Catalogue of Optically Visible Open Clusters and Candidates (Dias et al., 2002, 2003a), light grey, and the Catalogue modified with new data from Salaris et al. (2004), dark grey.

as representative of the error range for the entire sample, the youngest cluster (age $<0.5 \mathrm{Gyr}$ ) errors are very likely in the range 50-150 Myr with for older cluster errors in the range 150-250 Myr.

In order to test the reliability of the features found in the open cluster age distribution we have generated synthetic age distribution diagrams from the original data. Each simulation was composed of the following steps: 


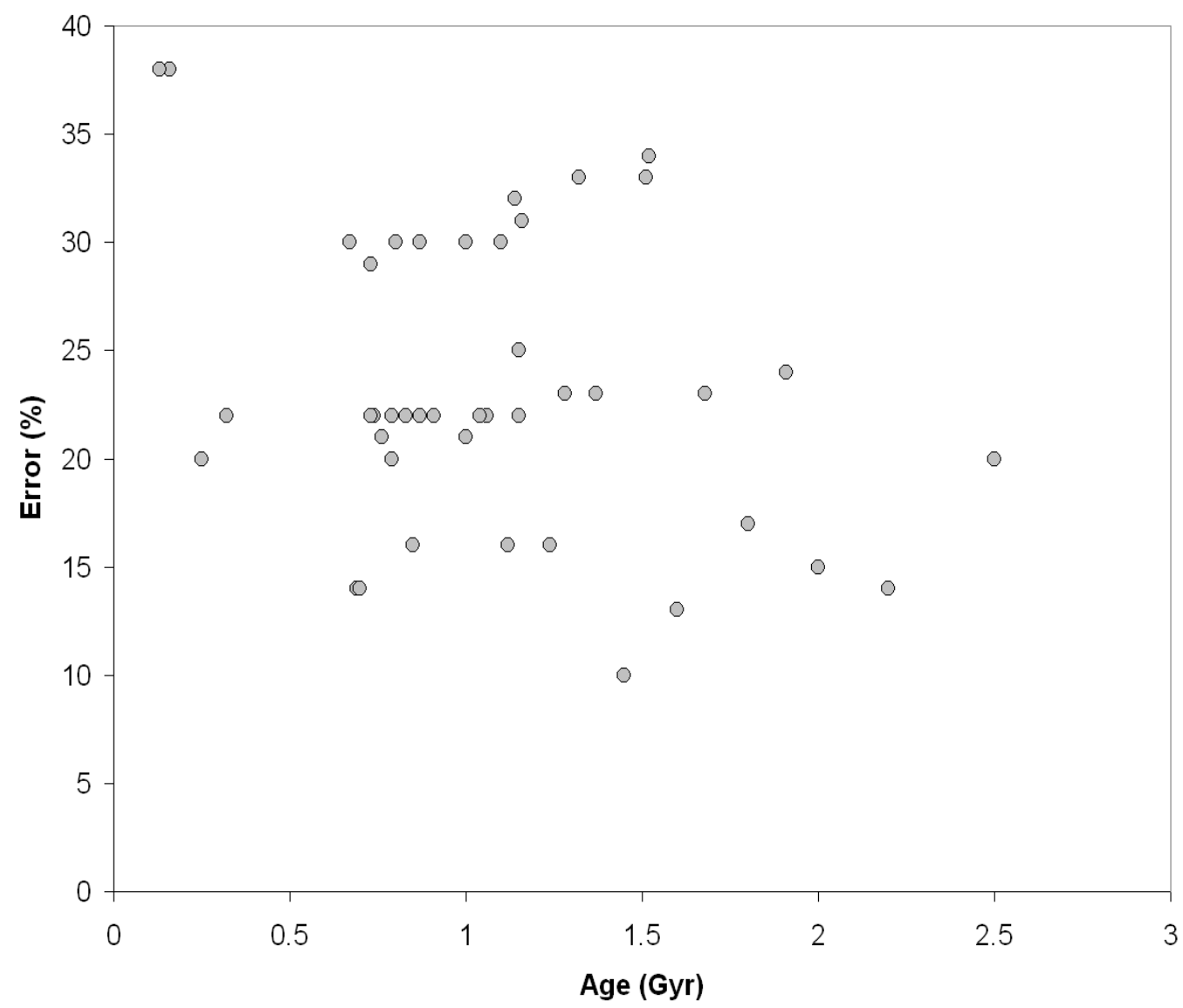

Fig. 5. Age errors as a function of the open cluster age for a selected sample of 52 objects with both age and error published (see the text). The average uncertainty is about $22 \%$ and the plot suggests that larger errors are associated with younger ages.

1) The original sample from Mermilliod (2003) is the input

2) A randomly distributed error within a given percentage of the input value is added/subtracted to each value in the original sample.

3) The simulated open cluster sample is binned at 0.050 Gyr intervals. In this way the real age of the open clusters in the sample is shifted randomly according to a given average error $(15,25$, and $35 \%)$.

4) The process is repeated 175,000 times per error value and we calculate the average number of objects per age bin. The final, averaged, sample is plotted in Fig. 6.

We have carried out three sets with 175,000 simulations to study this effect. These simulations appear to suggest that for age errors of about $15 \%$ or lower, most of the features in the age distribution remain relatively unaltered. For errors in age determination larger than about $25 \%$ only the youngest features 


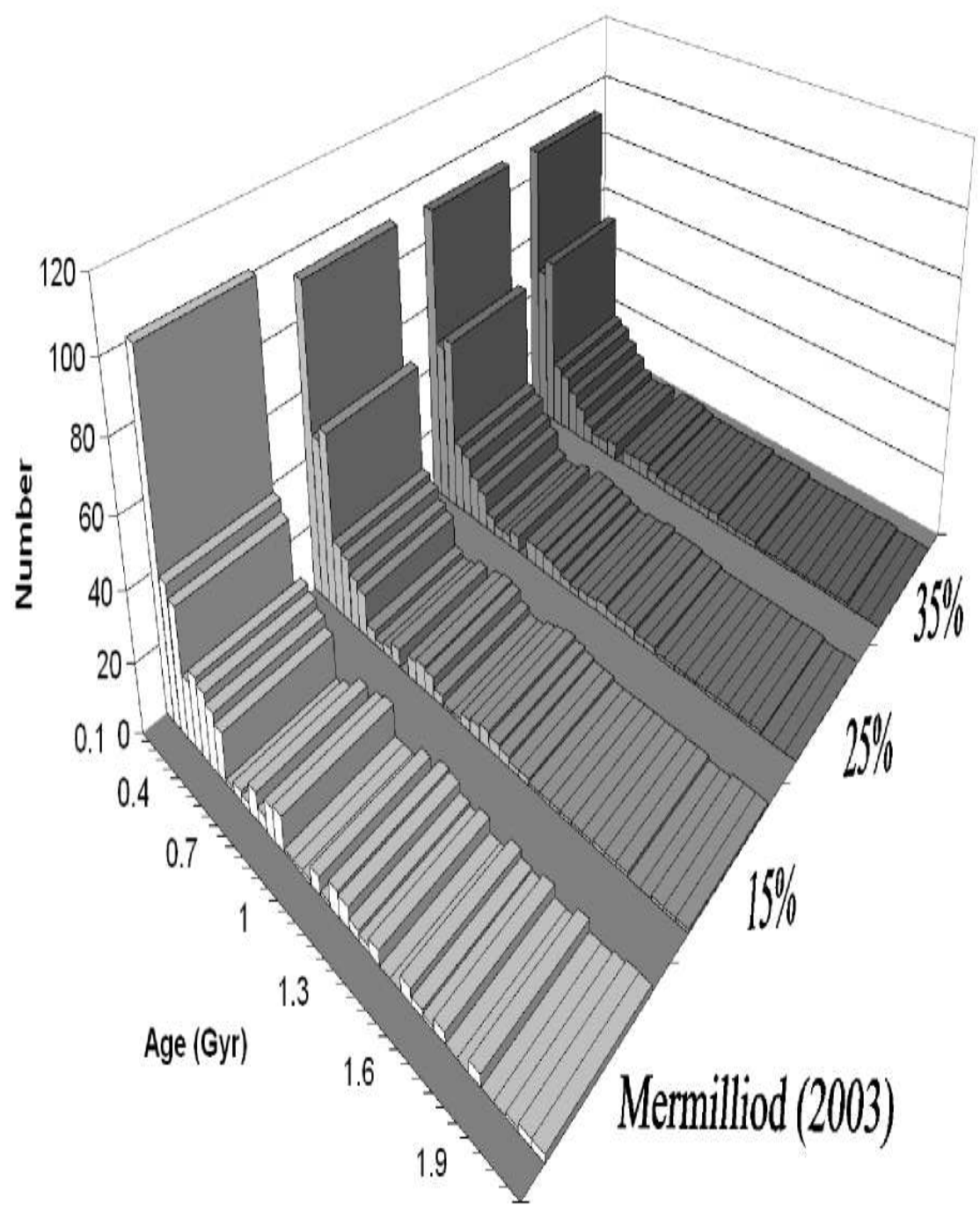

Fig. 6. Age distribution diagram of the open cluster sample from WEBDA. Each inner bar diagram gives the average of 175,000 simulated open cluster samples with different error range $(15 \%, 25 \%$, and $35 \%)$.

are recovered.

\section{The life span of open cluster models}

Fig. 4 is nothing more than the actual age distribution, a raw histogram of open cluster numbers vs. age, of relatively young, nearby open clusters. In order to interpret this distribution additional tools are needed. Experimental Stellar Dynamics may help us in this analysis by matching cluster dynamics and cluster data to provide a better view of the star formation history of the Milky Way disk. This section summarizes some relevant results on the evolution and dissolution of realistic $N$-body star cluster models. Some tech- 
nical details on the modeling process are also discussed as well as the initial mass functions used in the calculations. After an open cluster is formed and its massive stars have expelled the gas it will evolve steadily, losing stars on a relatively long time-scale. Distant encounters among cluster stars together with the Galactic tidal field cause the slow and gradual evaporation of the cluster. Close encounters and stellar evolution can speed up the process, specially for small, sparsely populated clusters. Primordial binaries are also very important (although less than stellar evolution) as they dominate completely the dynamical evolution of poor clusters. Violent, very fast cluster disruption can be induced during encounters with giant molecular clouds (Terlevich, 1983, 1985, 1987). The disintegration time-scale of open clusters depends on both population and Galactocentric distance; but, for a given Galactocentric distance, the initial population $\left(N_{o}\right)$ as well as the initial mass function are the main physical parameters to consider in order to analyze the long term evolution of the cluster.

The numerical results discussed here come from an extensive set of simulations (de la Fuente Marcos, 1995, 1996a, 1996b, 1997a, 1997b; de la Fuente Marcos \& de la Fuente Marcos, 2000, 2002) calculated with the standard $N$-body code NBODY5 (Aarseth, 1985, 1994, 1999, 2003) for clusters located in the solar neighbourhood. These calculations include the effects of stellar evolution, the Galactic tidal field, primordial binaries, and realistic initial mass functions (hereafter IMF). NBODY5 consists of a fourth-order predictor-corrector integration scheme with individual time steps. It utilizes an Ahmad-Cohen (1973) neighbour scheme to facilitate calculation of the gravitational forces, and handles close encounters via two-, three-, four-, and chain regularization techniques (Kustaanheimo \& Stiefel, 1965; Aarseth \& Zare, 1974; Mikkola, 1985; Mikkola \& Aarseth, 1993). Five different IMFs were used to generate stellar masses. Two standard power law models (Salpeter, 1995; Taff, 1974) and three modern IMFs due to the Miller \& Scalo (Miller \& Scalo, 1979; Eggleton et al., 1989), Kroupa (Kroupa \& Tout, 1992; Kroupa et al., 1990, 1991, 1993) and Scalo (Scalo, 1986). The IMFs used in our calculations differ mainly in the number of low-mass and high-mass stars generated. The Salpeter IMF is given reasonably well by the approximation

$$
\xi(M) \approx 0.03 M^{-2.35},
$$

where $\xi(M) \mathrm{d} M$ is the number of stars in the mass interval $M$ to $M+\mathrm{d} M$. The Taff IMF is given by

$$
\xi(M) \approx 0.03 M^{-\alpha},
$$

with $\alpha=2.50$ for clusters with $N \leq 100$ and $\alpha=2.65$ for clusters with $N>100$. For generating masses with the Miller \& Scalo IMF we use the 
function (Eggleton et al., 1989)

$$
M(\mathrm{X})=\frac{0.19 \mathrm{X}}{(1-\mathrm{X})^{0.75}+0.032(1-\mathrm{X})^{0.25}}
$$

where $\mathrm{X}$ is uniformly distributed in the interval $[0,1]$. For $\mathrm{X} \sim 0.7-0.999$, this gives a power-law expression with $\alpha=7 / 3$ and for $\mathrm{X}<<1$ it gives an approximately constant value. For $\mathrm{X}>0.999$, the IMF slope is increased to 5. The Kroupa IMF is given by (Kroupa et al., 1993)

$$
\xi(M)= \begin{cases}0.035 M^{-1.3} & \text { if } 0.08 \leq \mathrm{M}<0.5 \\ 0.019 M^{-2.2} & \text { if } 0.5 \leq \mathrm{M}<1.0 \\ 0.019 M^{-2.7} & \text { if } 1.0 \leq \mathrm{M}<\infty\end{cases}
$$

We use the mass-generating function

$$
M(\mathrm{X})=0.08+\frac{\gamma_{1} \mathrm{X}^{\gamma_{2}}+\gamma_{3} \mathrm{X}^{\gamma_{4}}}{(1-X)^{0.58}}
$$

as a convenient representation of these relations, where $\mathrm{X}$ is uniformly distributed in the interval $[0,1]$ and the $\gamma$-parameters are given by $\gamma_{1}=0.19$, $\gamma_{2}=1.55, \gamma_{3}=0.050$ and $\gamma_{4}=0.6$. No masses less than $0.08 M_{\odot}$ are generated by this formula. It is based on Scalo's (1986) initial mass function. For the Scalo IMF we use the mass-generating function

$$
M(\mathrm{X})=\frac{0.3 \mathrm{X}}{(1-X)^{0.55}}
$$

where $\mathrm{X}$ is uniformly distributed in the interval $[0,1]$. It is similar to Kroupa IMF except for $M<0.16$; Scalo IMF gives a smaller number of stars with those masses. The Kroupa IMF is the most recent IMF we have used, it generates a greater number of low-mass stars and a smaller number of heavy stars. On the contrary, the Salpeter IMF gives a smaller number of low-mass stars and a greater number of massive stars. Among these, the Taff IMF gives a smaller number of massive stars than the Salpeter one but greater than the Miller \& Scalo one. The Scalo IMF is intermediate between Miller \& Scalo and Kroupa IMFs. For low-mass stars, the Miller \& Scalo IMF gives a greater number than the Salpeter and Taff ones but smaller than Scalo and Kroupa IMFs. In addition, three different star densities (uniform, $\propto 1 / r^{2}$, Plummer) have been used to generate the initial positions of the stars in the cluster model. Since the initial membership of a star cluster is the main parameter with regard to cluster life-time for a given Galactocentric distance, stellar 
evolution (through the IMF) is the only process able to induce a differential behaviour as a function of $N$.

Averaging cluster life-times for the entire library of models as a function of $N$ to fit a power law to the data we obtain $\tau=0.011 N^{0.68}$ (in Gyr) with a correlation coefficient of $r=0.995$ (Fig, 7). This slope is very similar to the slopes found by Baumgardt (2001) and Baumgardt \& Makino (2003) although the details of our simulations are rather different. The above evolutionary track is only strictly valid for cluster models with $N>100-150$ members. Unfortunately, for smaller clusters the characteristic life-time is not very well defined as the energy released in a single supernova event may be larger that the binding energy of the entire cluster (de la Fuente Marcos, 1993). For clusters with $N<50$, life-times are in the range 5-40 Myr and for $N=75$, $\tau<80$. In general, the fluctuation range for the life-time of small- $N$ cluster models is very large and depends strongly on the initial mass function, the spatial distribution, and the binary fraction. For richer clusters, simulations indicate that, in the solar neighbourhood and neglecting interactions with giant molecular clouds and the effect of the cluster gas on the early stages of the evolution of the cluster, an open cluster has to include about 200-400 stars (at least) in order to survive for about 0.5 Gyr, 400-700 to last 0.7 Gyr, 700-1000 to be detectable after 0.9 Gyr and 1000-2000 to survive for about 1.3 Gyr. If a cluster is still observable after 2 Gyr, its initial population was at least 3000 stars. In terms of cluster masses and for an average stellar mass of $0.4 M_{\odot}$, the oldest open clusters considered in this paper $(2 \mathrm{Gyr})$ were born with masses in excess of $1.2 \times 10^{3} M_{\odot}$ and very likely around $2 \times 10^{3} M_{\odot}$.

The astrophysical interpretation of the solid line in Fig. 7 is rather clear: it is the open cluster death line. Open clusters well below the line are young and they evolve towards the line as they lose stars into the field. As it evolves, an average open cluster spends most of its life-time close to the death line moving parallel to it, in the population vs. age diagram. Open clusters become part of the stellar field when they cross the line. It is, however, very difficult to find open cluster memberships and masses among the published literature. Star counts are in many cases unreliable and proper motions are not always available to confirm membership. There is however an observational parameter that may help in open cluster membership determinations: the integrated magnitude of the cluster.

The integrated absolute visual magnitude of a star cluster can be written as:

$$
M_{v}(\text { cluster })-M_{v}(\operatorname{sun})=-2.5 \log \frac{L(\text { cluster })}{L(\text { sun })}
$$

where the absolute visual magnitude of the Sun is 4.82 (e.g. Zombeck, 1990) and the luminosity of the cluster could be, in principle, estimated using the 


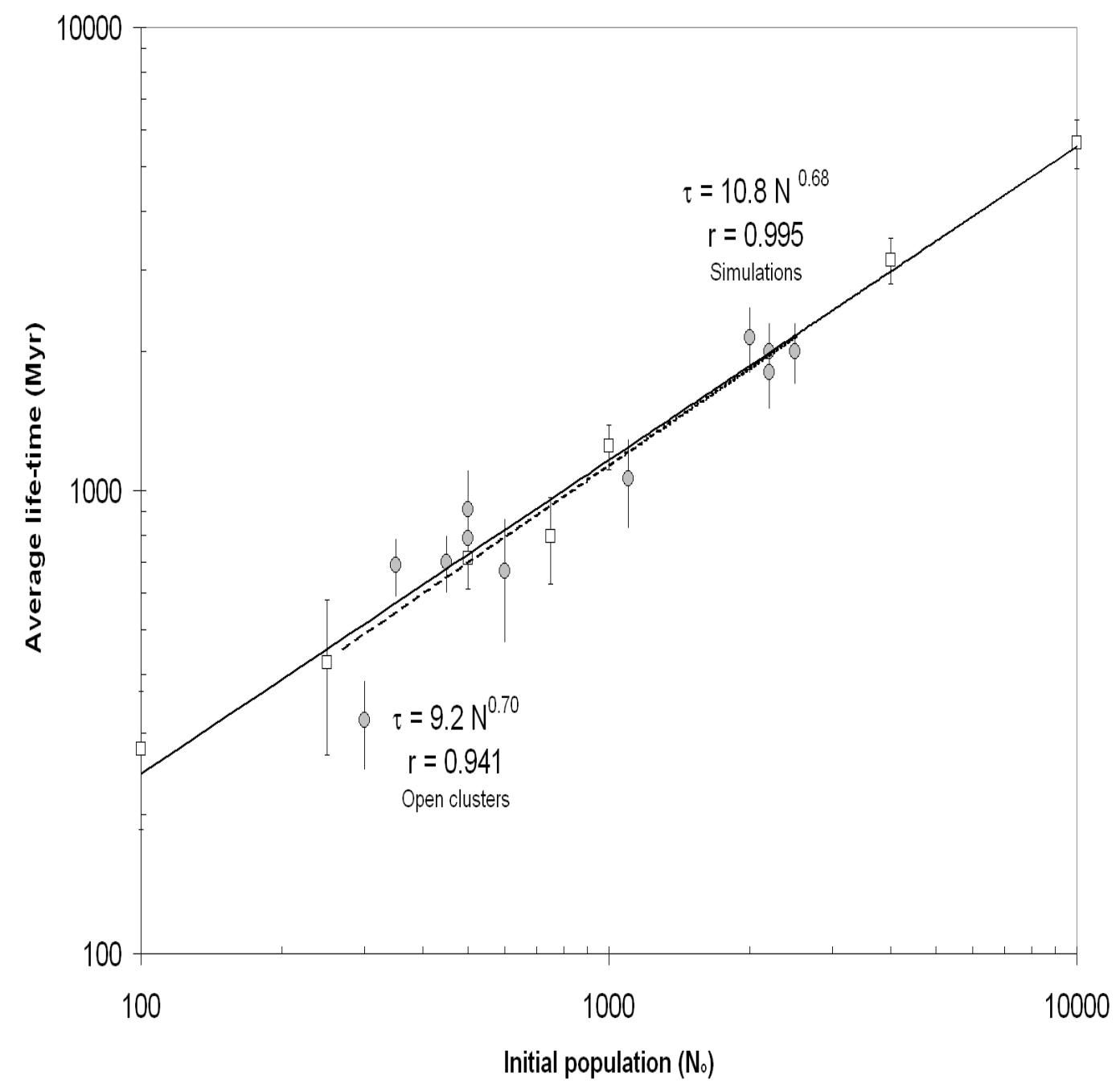

Fig. 7. Characteristic life-time $(\tau)$ as a function of the initial membership for a set of realistic open cluster models (de la Fuente Marcos 1995, 1996a, 1996b, 1997a, 1997b; de la Fuente Marcos \& de la Fuente Marcos 2000, 2002). The error bars indicate the standard deviation found for each value of $N$. The solid line represents the power law fit to the models data (empty squares), $\tau=10.8 N^{0.68}$ with a correlation coefficient of $r=0.995$. Actual open clusters are plotted as grey circles. The dashed line represents the power law fit to the open cluster data (grey circles), $\tau=9.2 N^{0.70}$ with a correlation coefficient of $r=0.941$. Open cluster memberships have been estimated using the model described in the text.

mass-luminosity relationship $L \propto M^{\beta}$, with $\beta \approx 3.8$ (Popper, 1980) for stars in the main sequence. Unfortunately, the main contribution to the intrinsic brightness of a relatively young cluster is not coming from the stars on the main sequence but from red (or blue) subgiants or giants. Taking this fact into account the luminosity of the cluster can be written as:

$$
L \text { (cluster) }=N_{N M S}<L_{N M S}>+N_{M S}<L_{M S}>
$$


where $N_{M S}$ and $N_{N M S}$ are the number of stars in the main sequence and outside the main sequence, respectively. Their relative contribution to the integrated luminosity of the cluster is obtained multiplying by the corresponding average luminosity. This average luminosity can be obtained from the average mass and the mass-luminosity relationship for the contribution from the main sequence. For subgiants or giants it is also possible to obtain an average luminosity using data for standard stars (e.g. Zombeck, 1990). In our calculations we will consider an average luminosity for stars outside the main sequence of $565 L_{\odot}$. Combining Eqs. 7, 8, $N=N_{M S}+N_{N M S}$, and considering the average stellar mass $M$ we obtain:

$$
N=\frac{1}{M^{3.8}+\frac{N_{N M S}}{N}<L_{N M S}\left(L_{\odot}\right)>} 10^{-\frac{\left(M_{v}(\text { cluster })-M_{v}(\mathrm{sun})\right)}{2.5}} .
$$

The fraction of stars outside the main sequence can be estimated assuming an IMF and taking into account that an estimation of the life-time in the main sequence for a given star of mass $M$ and luminosity $L$ is $\propto M / L$. The relationship given by Eq. 9 can be calibrated using a few clusters with well established memberships (e.g. Hyades, Pleiades). For the age range studied in this work the fraction of stars outside the main sequence is in the range $0.5-10 \%$. The final value of $N$ is affected by our choice for this fraction but the main contribution to the uncertainty in $N$ is coming from the observational determination of the integrated magnitude. For Galactic open clusters this uncertainty could be $\pm 0.5 \mathrm{mag}$ (Battinelli et al., 1994; Lata et al., 2002). This translates into $20 \%$ errors in $N$. We have applied this approach to estimate the current population of a small sample of open clusters (Hyades, Pleiades, IC 4756, NGC 2204, NGC 2506, NGC 7789, NGC 1245, NGC 381, NGC 2324, and NGC 2192) with age, error in age, and integrated absolute visual magnitude available from published sources (WEBDA; Battinelli et al., 1994; Gray, 1965; Pandey et al., 1989; Lata et al., 2002; Sagar et al., 1983; Spassova \& Beav, 1985). The results are also plotted in Fig. 7, the dashed line represents the power law fit to the open cluster data (grey circles), $\tau=9.2 N^{0.70}$ with a correlation coefficient of $r=0.941$. Only one open cluster younger than 500 Myr has been included because all of them are well below the limiting line. The power law fit includes all the plotted points. If the youngest cluster is not considered then the correlation coefficient is essentially 1 . In any case, Fig. 7 appears to confirm that observational data and results from $N$-body simulations are fully consistent within the error limits.

\section{Method and results}

In this section we provide a detailed description of our open cluster age dis-

tribution method. As stated in Section 2, our results are mainly sensitive to 
the errors in age determination but also to the degree of completeness of the open cluster sample. These two effects have been analyzed in Section 2 and are discussed in detail below. On the other hand, any errors in theoretical stellar models also propagate into the results obtained because the published open cluster ages always make reference to theoretical stellar models, sometimes through direct isochrone comparison, others through the use of morphological features found in the cluster color-magnitude diagram.

\subsection{Method and assumptions}

The open cluster catalogues offer high-quality data for a relatively large number of objects in the Milky Way disk, both in the solar neighbourhood and beyond. In particular, the age data can be used to construct an age distribution for the Milky Way open clusters, Fig. 4. Assuming that the sample considered is representative of the entire Galactic disk, the star formation rate can be derived from its age distribution, as the number of open clusters in each age bin is, by hypothesis, correlated with the number of open clusters initially born at that time. Once an age distribution is available, it is in principle easy to recover the star formation history that gave rise to the observed age distribution by using results from realistic $N$-body simulations. This method permits the reconstruction of the global star formation history of the Galactic disk with a time resolution of 0.050 Gyr over the last 2 Gyr. Our method does not assume any a priori structure or condition on the star formation rate and it basically consists of two steps: (i) Construct a representative sample of open clusters. In principle, the optimal approach will be to construct a volume-limited open cluster sample in the solar vicinity (see Section 6). The cluster sample spans a very large range of ages. (ii) Construct the age distribution diagram for the sample. (iii) Infer the star formation history from the diagram. Observations of bursts of star formation in other galaxies indicate that intense star formation is always associated with production of large star clusters. The life span of larger clusters is longer; therefore, an usually high number of open clusters at a given time interval can be interpreted as the result of an event of enhanced star formation at that given age. This is probably the main a priori assumption maintained throughout the paper although it appears to be strongly supported by observational evidence. If star cluster masses are sampled from an open cluster initial mass function, larger numbers translate into increased probability of formation of large star clusters. Luminous, and therefore rich, young star clusters are found whenever there is vigorous star formation, whether it be in galaxy mergers or starburst galaxies. Galaxies with very active star formation have proportionally more of their stars in clusters than in the field, with some of them devoting as much as $15-20 \%$ of their luminosity to clusters (Larsen \& Richtler, 2000). Presently available observational data strongly suggests that the cluster forming frequency is highest during 
violent bursts of star formation (van den Bergh, 2000).

\subsection{Results}

The resulting star formation history comes directly from the age distribution (Figs. 4, 8), in an approach which assumes that the most frequent ages of the open clusters indicate the epochs when the star formation was more intense if we take into account that star clusters are the elementary units of the star formation process. We have not included any volume correction because the number of clusters more distant than about $6 \mathrm{kpc}$ is negligible for the two samples considered. The volume effect as well as evolutionary corrections are considered in Section 6). We refer basically to the clumps of clusters at about $0.35,0.70,1.13,1.50$, and 1.93 Gyr. These clumps will be identified as burst $1,2,3,4$, and 5, respectively. The actual existence of burst 1, 2 and 3 is not significantly (see the detailed discussion below) affected by uncertainties in the cluster ages as errors are smaller than 0.3 Gyr for all clusters (younger than 1.2 Gyr) in the samples considered. However, for clusters older than 1.2 Gyr age errors are larger, therefore the statistical significance of bursts 4 and 5 is not, in principle, as well established as for 1, 2, and 3.

On the other hand, if we assume that the Galactic disk is not currently undergoing a burst of star formation and that the age distribution for stars younger than 0.2 Gyr is representative of the monotonic (quiescent) star formation rate in the Milky Way disk (i.e. it has not been polluted by an enhanced episode of star formation), then the fraction of open clusters that survives for up to about 0.25 Gyr is about $92 \%$. This corresponds to the characteristic life-time of clusters with $N \leq 150$. In other words, observations indicate that only $8 \%$ of all the open clusters are born with stellar populations larger than about 200 stars or masses $>80 M_{\odot}$. This feature can be interpreted as implying that star formation takes place preferentially in clusters of that size. This result, that most of the stars appear to have been formed in small star clusters, has already been pointed out by Kroupa (1995a, b, c) and de la Fuente Marcos (1997b). However, a single 3,000 stars open cluster can produce as many field stars as 60 small (50 stars) clusters and, as pointed out above, the number of large clusters violently destroyed during cluster-giant molecular cloud interactions, even though difficult to estimate, can be very significant. On the other hand, during an episode of enhanced star formation even much larger clusters (up to 40,000 stars) can be formed. Therefore it may also be possible that most of the field stars have been formed in large clusters, however we do not observe many large clusters today and the previous possibility remains rather speculative. In any case, the cumulative age distribution for the full open cluster sample shows a departure from the predictions of constant formation rate and exponentially declining dissolution rate. 


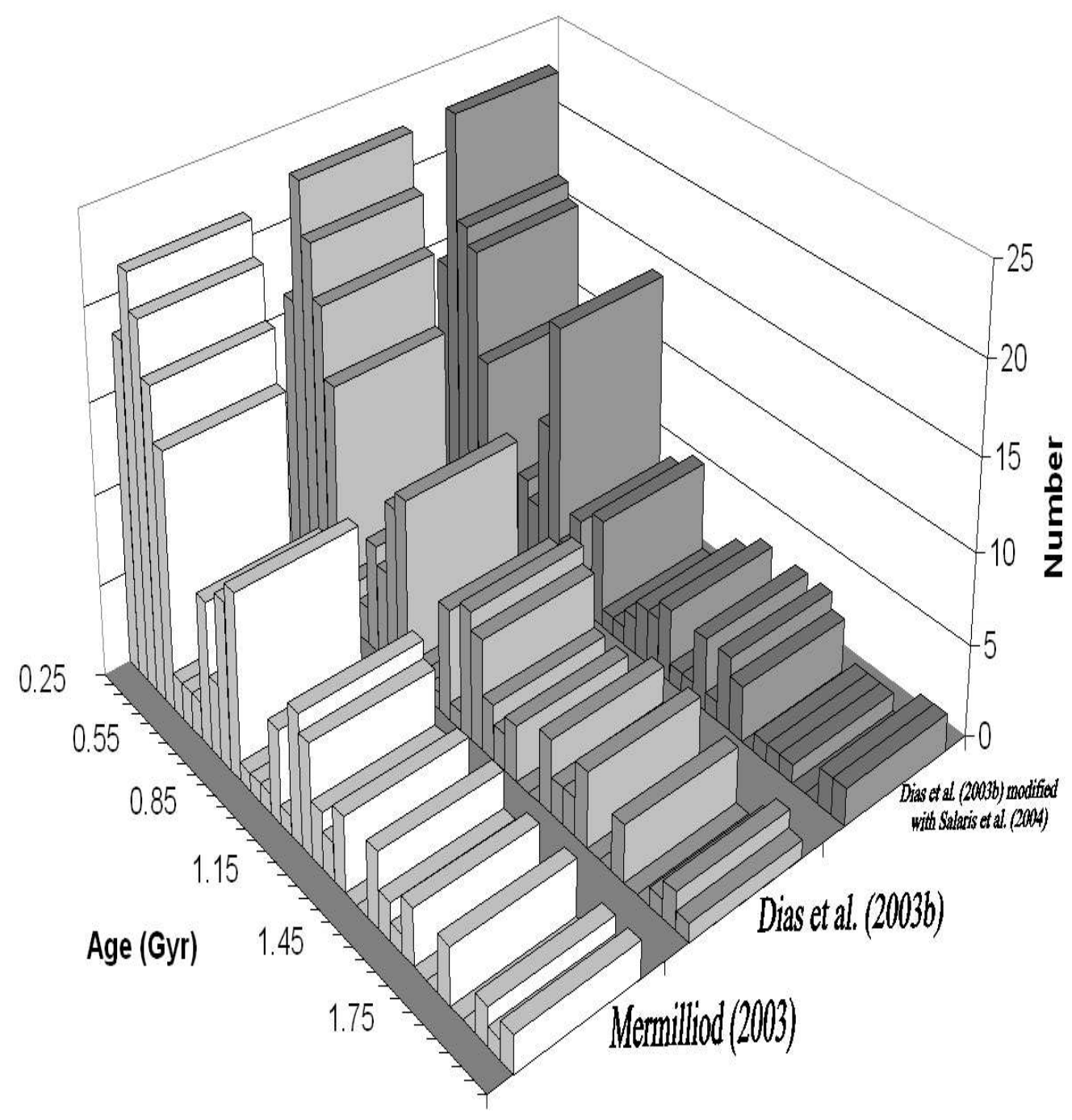

Fig. 8. Detail of Fig. 4 for the age range 0.2-2.0 Gyr, same bin size.

\subsection{Quiescent vs. burst of star formation}

Assuming that the Galactic disk has never been polluted by any extragalactic stellar populations, our previous analysis suggests that superimposed on a relatively small level of quiescent star formation, mainly in small- $N$ star clusters, the star formation rate has experienced at least 5 episodes of enhanced star formation lasting about 0.2 Gyr each, with production of larger clusters. This cyclic behavior appears to show a period of $0.4 \pm 0.1$ Gyr. Although the observed cyclic behavior can be easily explained as triggered by density waves (Hernandez et al., 2000a; Martos et al., 2004), the analysis in this section is also exploring the possibility of periodic, tidally triggered star formation bursts as an alternative (or possibly concurrent) scenario to explain the enhancements observed in the open cluster age distribution. There is evidence for a recent pericentric passage of the Sagittarius Dwarf Spheroidal Galaxy 
$\sim 50$ Myr ago (Johnston et al., 1999). If this close interaction was able to tidally trigger star formation in the disk of the Milky Way, its effects should currently be observable and it could be considered as burst 0 . There are in fact observational signatures of unusually rich young open clusters (or even protoglobulars) in the disk of the Milky Way: e.g. Cyg OB2 (Knödlseder, 2000; Knödlseder et al., 2002; Comerón et al., 2002; Hanson, 2003). We will however neglect this possibility in our subsequent discussion.

\subsubsection{The 0.35 Gyr burst}

The most recent star formation bursts are also the most likely bursts to have occurred, since they took place in the very recent past, and so are less affected by the age errors. Unfortunately, our analysis in Section 2 appears to suggest that age errors are larger for younger clusters. The youngest (0.25-0.45 Gyr) burst found in this work appears around the same age in all the three samples, lasting $0.2 \mathrm{Gyr}$ that is the typical duration of bursts found in previous work. This burst is temporally coincident with a perigalactic passage of the Small Magellanic Cloud (hereafter SMC) with the Milky Way as predicted by Lin et al. (1995). Harris and Zaritsky (2004) have studied the star formation history of the SMC and have found four major bursts of star formation: 0.06, 0.4, 2.5, and 8.4 Gyr. Models by Zaritsky and Harris (2004) indicate that the 0.4 Gyr peak in the SMC stellar age function coincides with one of the epochs of closest approach between SMC and the Milky Way. During the interaction, the elevation in the star formation rate was significant (> factor of 2) over the quiescent rate of star formation in the SMC.

\subsubsection{The 0.70 Gyr burst}

This burst appears at 0.6-0.8 Gyr, lasting also 0.2 Gyr, in WEBDA and NCOVOCC but appears to be wider, 0.6-1.0 Gyr, for the sample including Salaris et al. (2004), shifting the peak to 0.8 Gyr. Johnston et al. (1999) have found that the Sagittarius Dwarf Galaxy had a pericentric passage $\sim 0.7 \mathrm{Gyr}$ ago. This close interaction is temporally coincident with burst 2 .

\subsubsection{The 1.13 Gyr burst}

From WEBDA, this burst started 1.3 Gyr ago, lasting about 350 Myr. However, NCOVOCC gives the same starting age but lasting $0.3 \mathrm{Gyr}$. The sample corrected with Salaris et al. (2004) data differs also only in the duration, 0.2 Gyr. This burst is temporally coincident with a perigalactic passage of the SMC with the Milky Way (Lin et al., 1995). 


\subsubsection{The 1.50 Gyr burst}

The three samples indicate that the burst started 1.6 Gyr ago, lasting 0.2 Gyr. This burst appears to be coincident with a close encounter between the Magellanic Clouds and the Galaxy (Murai \& Fujimoto, 1980; Gardiner et al., 1994; Lin et al., 1995). On the other hand, the Sagittarius Dwarf Galaxy had another pericentric passage around the same time (Johnston, 1998; Johnston et al., 1999).

\subsubsection{The 1.93 Gyr burst}

This is the oldest star formation burst identified in this work and it is also the most affected by the age errors. WEBDA and NCOVOCC indicate that this burst started 2 Gyr ago, lasting 0.15 Gyr. The third sample suggests a burst lasting $0.1 \mathrm{Gyr}$. This is the only burst that does not appear to coincide with any predicted or modelled interaction with a neighbour galaxy.

In spite of the time coincidences, interpreting the observed periodicity in the age distribution of young, nearby open clusters as evidence in support of a tidally triggered star formation history is somewhat speculative. On the other hand, a close interaction with the Magellanic Clouds cannot be compared dynamically to that of the Sagittarius or the Canis Majoris dwarf galaxies.

Using our method without any corrections it is, however, difficult to estimate the evolution of the absolute star formation rate with the age of the disk. Nevertheless, it is possible to attempt a rough estimate: the number of clusters in bursts $1,2,3,4$, and 5 from the WEBDA sample is $73,31,28,10$, and 5 respectively, for the Dias et al. sample is $73,30,28,12$, and 4 and for Salaris et al. is $66,49,12,12$, and 4. Data seem to suggest that for WEBDA and Dias et al. burst 2 was likely the strongest. If under quiescent star formation only $2 \%$ of open clusters survive for more than 2 Gyr, during burst 2 the star formation increased by at least a factor 4 . Salaris et al. data also indicate that burst 2 was the most important, followed by burst 4 . During burst 2 the number of open clusters formed increased by a factor 8 at least.

\subsection{Limitations}

As pointed out before, the star formation history inferred for the Milky Way disk in the previous section is affected by selection effects and cluster age errors, therefore some features in the derived age distribution could be caused by the incompleteness of the sample. As a result of these limitations, the actual amplitude of the bursts is likely higher. Working with a flux-limited sample makes it very difficult to estimate the evolution of the absolute star formation 
rate with the age of the disk. Nevertheless, in this section we try to assess the accuracy of our results.

The age errors affect considerably the duration of the star formation events, since they tend to scatter the ages of the star clusters originally born in a burst. We can expect that this error could smear out peaks and fill in gaps in the age distribution. In Section 2, we have tested our results using synthetic age distribution diagrams from the original data (see Fig. 6). Simulations appear to indicate that for age errors of about $15 \%$ or lower bursts 2,3 , 4, and 5 remain relatively unaltered both in terms of age and duration, however burst 1 seems to split into two bursts, one at 0.2 Gyr and another one at 0.5 Gyr. For errors in age determination larger than about $25 \%$ only bursts at $0.2,0.5$, and 0.7 are recovered. However, our error analysis in Section 2 appears to indicate that the older the cluster, the lower the error. Therefore it could be possible that errors for clusters older than 1.5 Gyr are in the range 15-25\% and the five bursts can be recovered.

Our derivation of the star formation history of the Milky Way disk uses a sample of dynamically bound, disk star clusters or open clusters. This sample is assumed as not polluted by unbound star clusters or associations. However, associations dissolve on a time-scale of $\sim 50$ Myr so even if some degree of contamination remains, the contribution to the final conclusions is negligible. It is only significant for the first bin in our age distribution diagram. On the other hand, it is known that a typical cluster in the outer disk will survive about twice as long as one in the inner disk (Janes et al., 1988). The outer disk group is an homogeneous group whose members are dissolving on a time-scale on the order of 3-4 Gyr. This contribution is however negligible as we restrict our analysis to clusters younger than 2 Gyr. In our previous analysis we have assumed that this age distribution is depopulated from extragalactic objects, so no open clusters have been incorporated into the galactic disk from accreted galaxies. An alternative, plausible but rather speculative scenario with excess clusters coming from accreted dwarf galaxies is considered in Section 8.

Limitations on the applicability of our method to a wider time range appear in connection with the small number of known open clusters older than 2 Gyr. With a resolution of 0.05 Gyr, our results are not statistically significant for an age older than 2 Gyr as the number of objects per age bin is too low. The need to provide reliable results limits the age range over which we can derive the star formation history to 0-2 Gyr, with the time resolution considered throughout the paper. However, our method can be applied to a wider time range if the time resolution is lowered (de la Fuente Marcos \& de la Fuente Marcos, 2004). 


\section{The last 0.2 Gyr}

Contrary to what is sometimes assumed in the early stages of the star formation history of the Milky Way disk, which were ruled by accretion events and early mergers of satellite dwarf galaxies and star clusters that contributed to the formation of a substantial fraction of the old and metal-deficient stars in the Galactic disk, during the last 0.2 Gyr the star formation rate (see Fig. 9) has been remarkably stable with apparently no events of enhanced star formation.

Fig. 9 appears to indicate that the open cluster sample younger than 0.2 Gyr can be considered representative in the analysis of quiescent star formation in the Milky Way disk. The characteristic time-scale to assemble and give birth to an open cluster seems to be $\sim 10$ Myr with (at least) about 80 new open clusters being formed every 10 Myr or 8 clusters/Myr within about $3 \mathrm{kpc}$ from the Sun. About $80 \%$ of newly formed clusters are destroyed during the first 20 Myr of life.

For a time resolution of 5 Myr (Fig. 9, small panel) a very interesting feature is visible: clusters younger than $5 \mathrm{Myr}$ are scarce. In principle, this feature may be interpreted as a minimum in the star formation rate, however young clusters are still embedded in their parent molecular cloud and this makes them difficult to detect. Dust obscuration appears to be the natural explanation for the origin of this feature. On the other hand, there is a sharp decrease in the number of open clusters observed for ages older than 15-20 Myr. There is an initial very rapid rate of cluster dissolution, gradually declining thereafter. Again the most simple interpretation is to assume that an enhanced star formation period started $\sim 20$ Myr ago. However, this feature can be better explained if we consider stellar evolution in open clusters. For a metallicity $Z$ $=0.02$, stellar evolution models (e.g. Schaller et al., 1992; Bressan et al., 1993) indicate that stars with masses above $10 M_{\odot}$ and older than about $20 \mathrm{Myr}$ have already exploded as supernovae. For a small cluster $(N<75)$, the energy released during one supernova event can be equivalent to the binding energy of the entire cluster. Therefore, early supernovae appear to be responsible for the violent destruction of about $80 \%$ of young open clusters.

In the following section, we use this information to generate a power-law open cluster initial mass function with slope -2.7 .

\subsection{Open Cluster Initial Mass Function}

The frequency distribution of stellar masses at birth, the so-called stellar initial mass function (hereafter SIMF), is a fundamental parameter to study the 


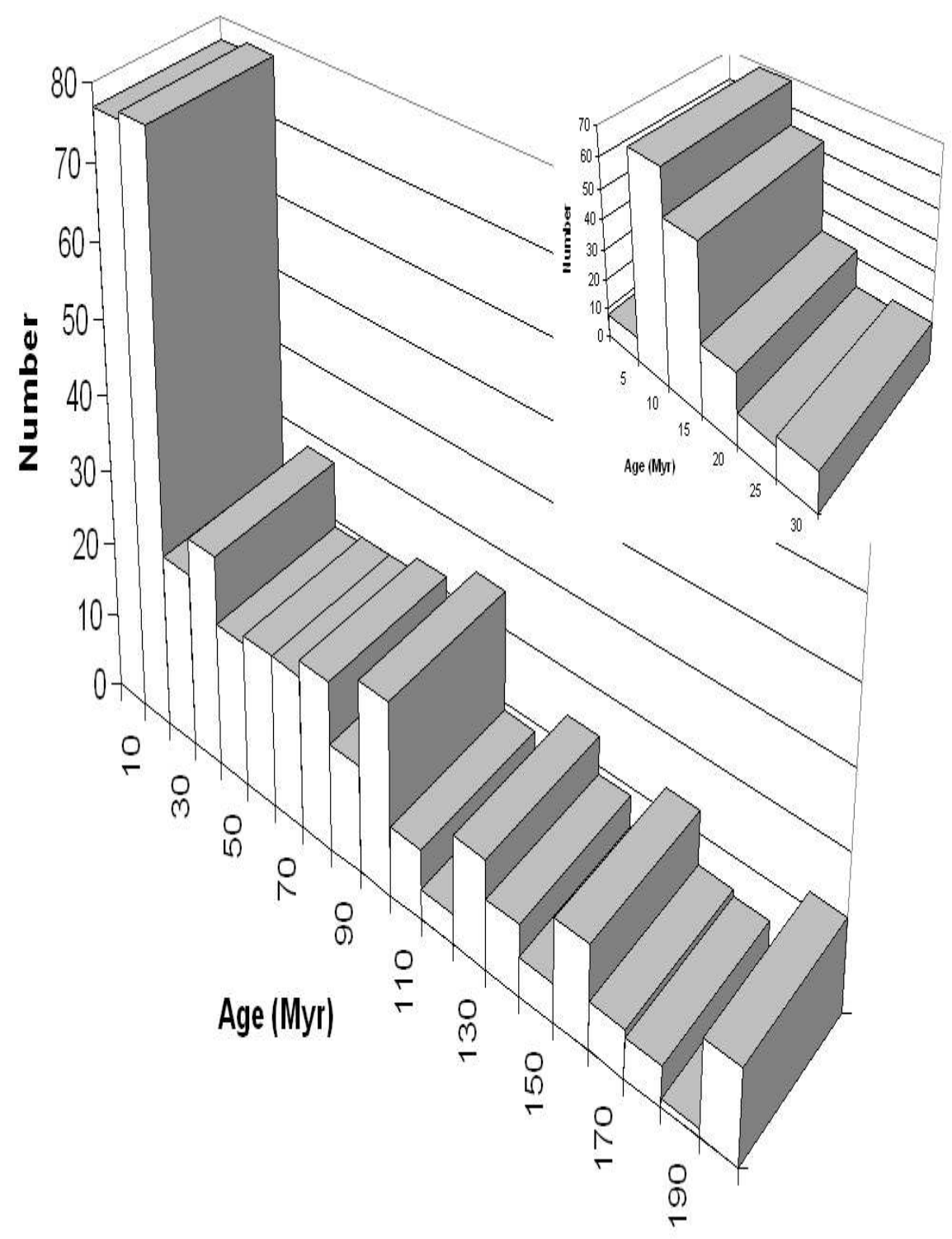

Fig. 9. Open cluster age distribution from one of the last update (October 2003) of WEBDA, for the last $0.2 \mathrm{Gyr}$ (main panel) with a time resolution of 0.01 Gyr. In the small panel we display the last 0.03 Gyr with a resolution of 0.005 Gyr.

stellar mass spectrum. Salpeter (1955) used the observed luminosity function for the solar neighbourhood and theoretical evolutionary times to derive a SIMF which may be approximated by a power-law:

$$
n(m) \propto m^{-\alpha}
$$

where $n(m)$ is the number of stars per unit mass interval. The original value of $\alpha$ found by Salpeter is 2.35, for masses between 0.4 and $10.0 M_{\odot}$. The determination of the SIMF is a cornerstone in Astrophysics, since the SIMF 
determines the evolution, surface brightness, chemical enrichment, and baryonic content of all galaxies and it is also a fundamental link between stellar and galactic evolution. For recent reviews on the SIMF see Kroupa (2002) and Chabrier (2003).

As many of the stars in the disk of the Milky Way appear to be the result of a clustered mode of star formation, the open cluster initial mass function (hereafter OCIMF) is also a fundamentally important distribution function to study cluster formation, chemical evolution of galaxies, and star formation in general. The fact that the SIMF and the OCIMF can be intimately related was first pointed out by Reddish (1978). However, deriving the OCIMF is very difficult as cluster mass determinations are affected by several observational and theoretical biases: obscuration by dust, flux limits, incomplete sampling of cluster haloes, lack of information on the evolutionary state of the cluster, and uncertainties in stellar models and cluster dissolution rates. Although the OCIMF is not well constrained it can be written as a power law of the type

$$
N(M) d(M) \sim M^{-\alpha} d M
$$

for $M_{\min }<M<M_{\max }, N(M)$ is the number of open clusters per unit mass interval. The cumulative distribution will be

$$
f(M) \sim M^{-\alpha+1}
$$

If the process by which open clusters are formed is also able to produce individual stars, it appears reasonable that both SIMF and OCIMF should show similar slopes within the observational errors. Reddish (1978) found a slope equal to -2.2 from a sample of 72 open clusters. Vázquez and Feinstein (1989) estimated the OCIMF for the Milky Way disk using a sample of 130 clusters from Lyngå's (1987) catalogue. In order to find the distribution, they used the values of the masses quoted in the catalogue to obtain a slope $\alpha=2.74 \pm 0.09$ for all the clusters in their sample. They also found that the slope is different for clusters with Galactocentric distances smaller than the Sun's and clusters with larger Galactocentric distances, although this may be an artifact due to incompleteness of the data. The topic of the distribution of the masses of star clusters has been re-visited for various star-forming galaxies (e.g. Bik et al., 2003; Elmegreen et al., 2000; Whitmore et al., 1999). Kroupa and Boily (2002) have recently approached the subject from a theoretical point of view focusing mainly on large clusters. They found that the entire Galactic population II stellar spheroid can be generated if star formation proceeded via embedded clusters distributed like a power-law mass function with slope in the range $(1.9,3.6)$. 
In this section we will infer the OCIMF for the quiescent Milky Way disk without considering the values of the cluster masses provided by the catalogues but taking into account the life-time scale obtained from simulations in Section 3 and the open cluster sample described in Section 2 to derive the OCIMF for the Milky Way disk. This method to construct an OCIMF has been outlined in Kroupa and Boily (2002). In Section 4.2 it has been evidenced that the disk of the Milky Way may have experienced several episodes of enhanced star formation over the last $2 \mathrm{Gyr}$, this implies that the OCIMF cannot be derived from the total sample of clusters, because the starburst will bias the OCIMF towards massive clusters. However, if we restrict our analysis to clusters with ages less than about $200 \mathrm{Myr}$, these clusters can be used to derive the OCIMF. This choice implies that the star formation rate has remained almost constant during the last $200 \mathrm{Myr}$; this assumption appears to be reasonably supported by our previous analysis. For an average stellar mass of $0.4 M_{\odot}$, we obtain that $\sim 65 \%$ of clusters have masses $\leq 20 M_{\odot}$, about $17 \%$ with $20 M_{\odot}<M<40 M_{\odot}$, $10 \%$ with $40 M_{\odot} \leq M<60 M_{\odot}, 6 \%$ with $60 M_{\odot}<M<75 M_{\odot}$, and $\sim 2 \%$ have masses $>75 M_{\odot}$, with a likely average of $150 M_{\odot}$. Observations and simulations seem to suggest that $M_{\min } \sim 2 M_{\odot}\left(N_{\min }=5\right)$ and $M_{\max } \sim 16,000 M_{\odot}$ $\left(N_{\max }=40,000\right)$. These results are consistent with an open cluster population being sampled from a power-law OCIMF with $\alpha \approx 2.7$ with a correlation coefficient $r=0.99$ (see Fig. 10). The error associated with this determination can be estimated from the standard deviation obtained for the life-time of simulated clusters and is $\sim 20 \%$. In this analysis we are not taking into consideration that a number of clusters (those younger than $\sim 60 \mathrm{Myr}$ ) may be still in the evolutionary stage driven by gas expulsion. This simplification is likely to have a minor impact on the overall results from this section because our results indicate that a significant fraction of open clusters formed during quiescence are in fact small and therefore short-lived.

\section{From the age distribution to the star formation history}

In the previous sections we have assumed that the open cluster sample under study is representative of the entire Galactic disk, therefore the evolution of the star formation rate can be inferred from its age distribution, since the number of open clusters in each age bin has to be correlated with the number of objects initially at that time as a result of dissolution processes. The most reliable transformation of the open cluster age distribution into history of the star formation rate comprises two intermediate corrections, namely the volume and evolutionary corrections. As stated in Section 4, a scale height correction can be neglected because the studied age range is $<2$ Gyr. The applied corrections are explained in the following sections. 


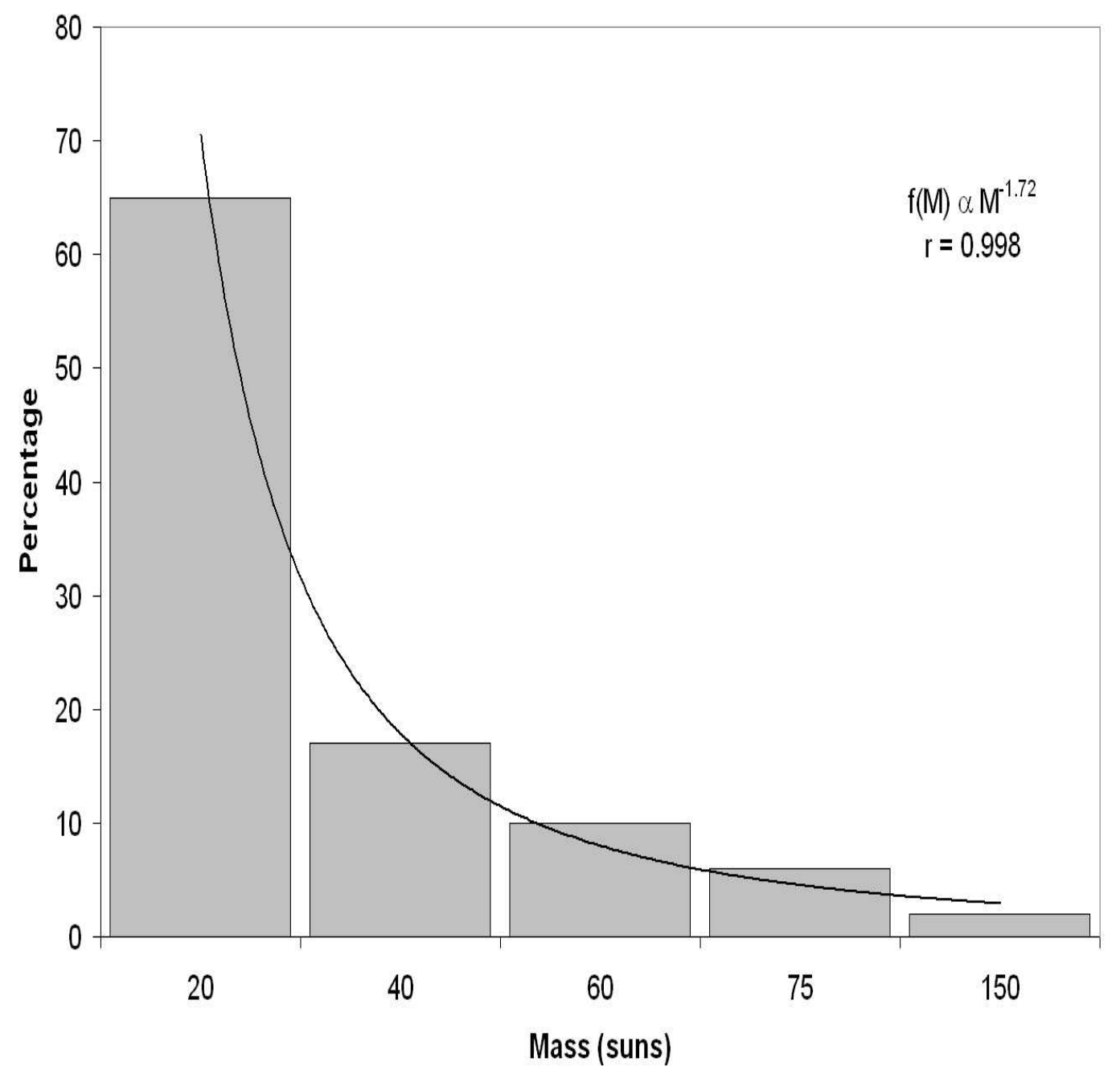

Fig. 10. Frequency distribution of the masses of open clusters. The OCIMF has been constructed from a representative sample of open clusters of known age fitting the age to star cluster evolution tracks from $N$-body calculations to infer initial cluster masses and creating the distribution from the ensemble of masses obtained.

\subsection{Evolutionary correction}

As pointed out in Section 3, a correction due to the dynamical evolution of the cluster (cluster disintegration) is needed because our sample includes clusters with different initial populations $\left(N_{o}\right)$. The more massive clusters have a life expectancy higher than the short-lived, small- $N$ clusters, thus the latter would be missing in the older age bins. It is however possible to correct for this effect using the OCIMF obtained in the previous section as well as the results from Section 3.

The corrections are given by the following formalism. The number of clusters 


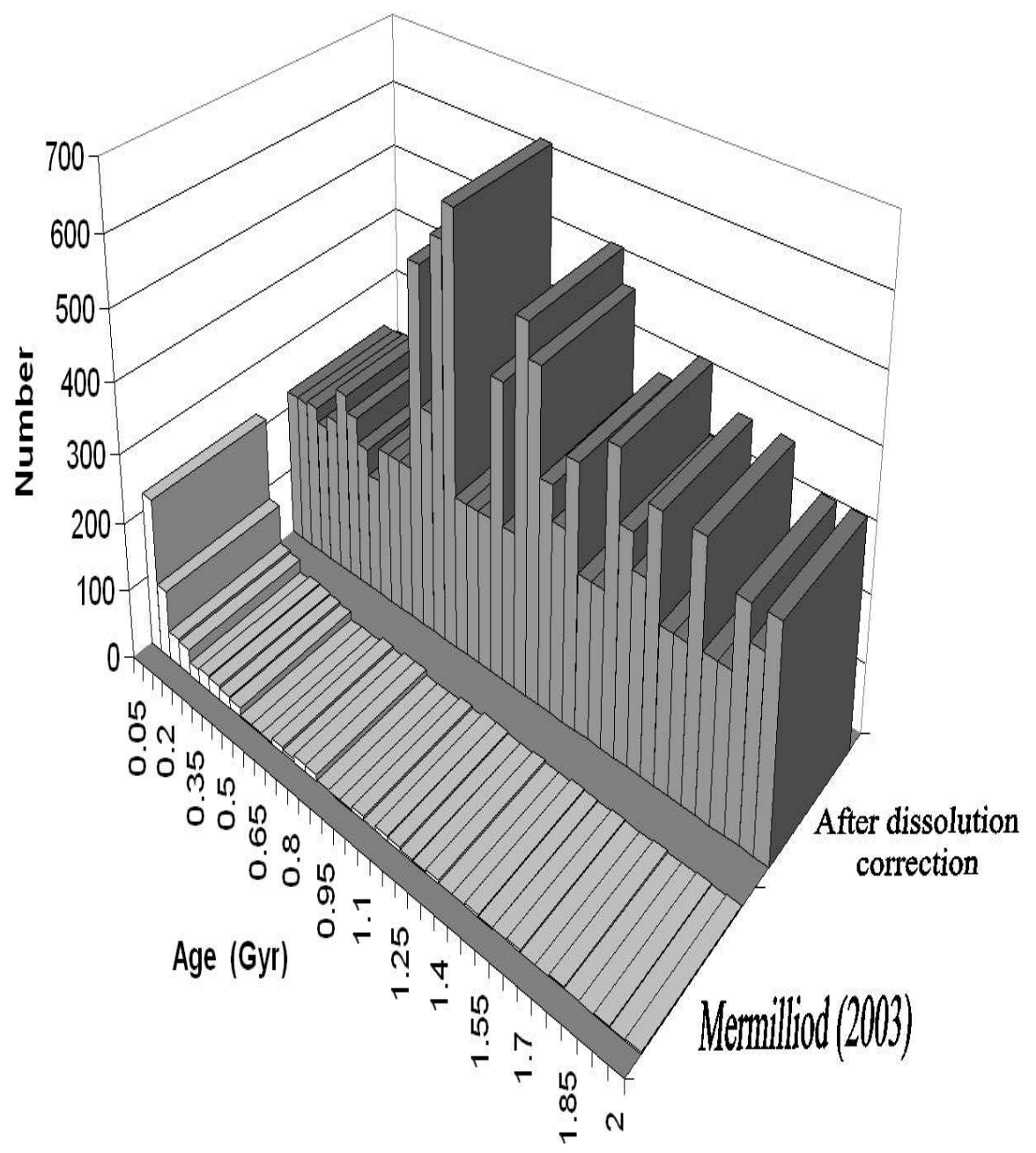

Fig. 11. Star formation history after the dissolution corrections (see the text), compared to the age histogram used throughout this paper.

born a time $t$ ago ( $t=0$ is present time) is the current number observed at age $t$ divided by the fraction of surviving clusters expected for an age $t$, $f$. The fraction of surviving clusters changes with $t$, therefore the life-time scale obtained in Section 3 is used to estimate the correct fraction of surviving clusters to be applied. In detail: for the second bin we have corrected by the same number in bin 1; bins 3-4 have been corrected using $f=0.18 ; f=$ 0.08 has been used in bins 5-9; bins 10-16 have been corrected by $f=0.02$. After $0.8 \mathrm{Gyr}$, we have assumed that no cluster formed during quiescent star formation epochs may have survived; therefore, the correction is made in a slightly different way: we add the reference level at $t=0$ to the standard correction with $f=0.02$. The age histogram from WEBDA and the corrected distribution appear in Fig. 11. The star formation history outlined in Section 4 is somewhat altered: 


\subsubsection{The 0.35 Gyr burst}

This burst appears at $0.20-0.35$ Gyr and it seems to be not very prominent.

\subsubsection{The 0.70 Gyr burst}

This burst appears at 0.6-0.8 Gyr, lasting also 0.2 Gyr. It appears to be much stronger than burst 1 .

\subsubsection{The 1.13 Gyr burst}

It started 1.3 Gyr ago and lasted 0.35 Gyr. It was the strongest. The unusually high level of enhanced star formation could be the result of the combined action of density waves and a strong tidal interaction with the SMC.

\subsubsection{The 1.50 Gyr burst}

This burst appears at 1.4-1.6 Gyr, lasting also 0.2 Gyr. It appears to be similar in strength to burst 2 .

\subsubsection{The 1.93 Gyr burst}

This burst started 2 Gyr ago, lasting 0.15 Gyr. Its activity was lower than for bursts 2 and 4 but much higher than 1 .

\subsection{Volume correction: the solar neighbourhood}

Since our original open cluster sample is not volume-limited, there could be a bias in the relative number of objects in each age bin: open clusters with different initial populations and metallicities have different integrated luminosities, thus the volume of space sampled varies from cluster to cluster. In principle, our method will produce optimal results for volume-limited open cluster samples. In this section we consider a cluster sample in the solar neighbourhood. The solar neighbourhood is defined as a volume centered on the Sun that is much smaller than the overall size of the Milky Way galaxy and yet large enough to contain a statistically useful sample of stars (see, e.g., Binney \& Tremaine, 1987). The appropriate size of the volume depends on which stars or objects are going to be investigated: for white dwarfs, which are both common and faint, it may consist of a sphere of radius 10 pc centred on the Sun, while for the bright but rare $\mathrm{O}$ and $\mathrm{B}$ stars, the solar neighbourhood may be 


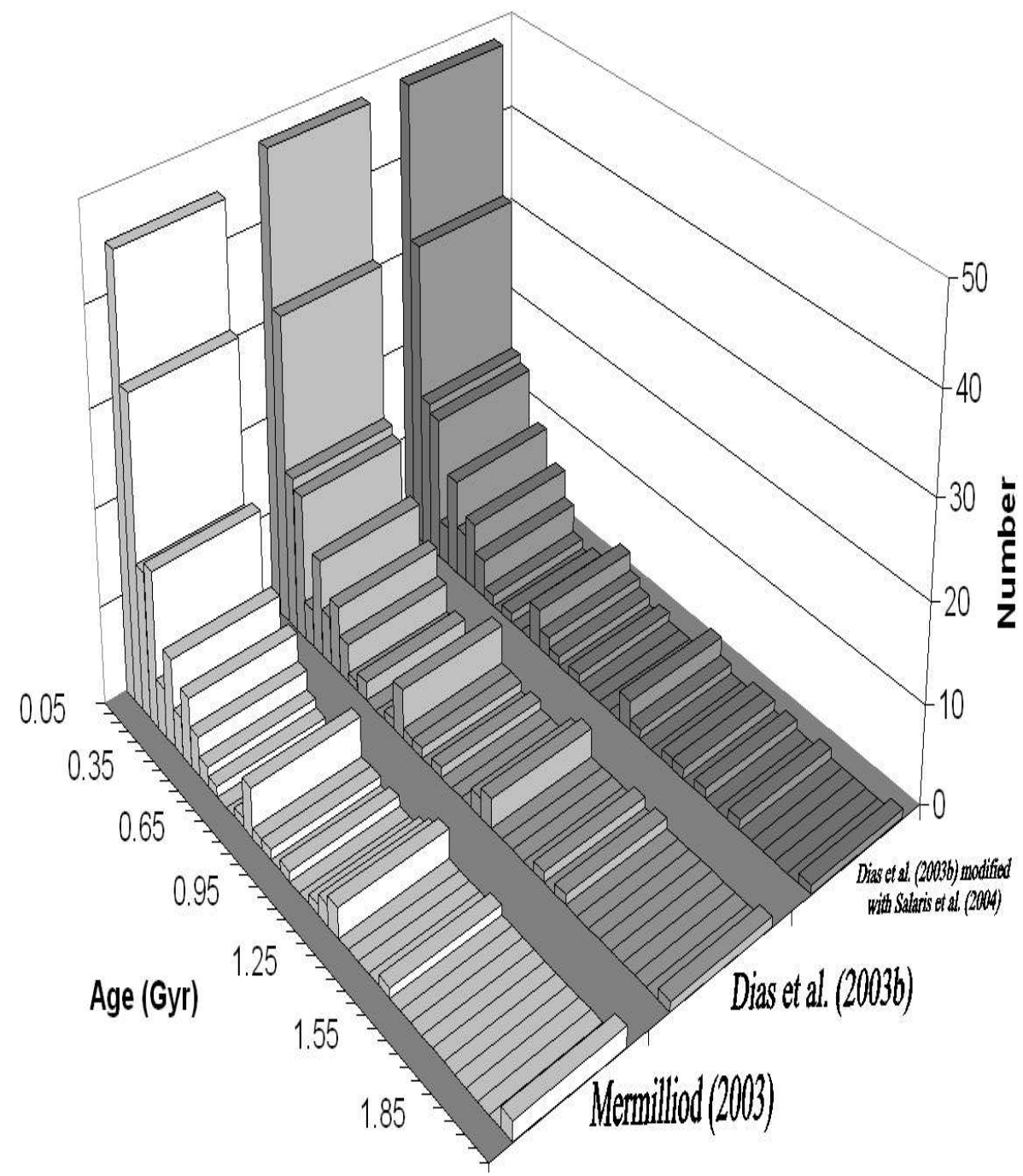

Fig. 12. Open cluster age distribution for objects in the three samples within $1 \mathrm{kpc}$ from the Sun, volume-limited sample.

considered to extend as far as $1 \mathrm{kpc}$ from the Sun. In our analysis we will consider the latter in order to retain a statistically significant sample of open clusters. There are 161 open clusters in our sample within $1 \mathrm{kpc}$ of the Sun (and younger than $2 \mathrm{Gyr}$ ).

If we restrict our analysis to clusters closer than $1 \mathrm{kpc}$, the age distribution plotted in Fig. 12 is obtained. Burst 1 is clearly identified at an age of 0.35 Gyr with a duration of about 0.2 Gyr. Burst 2 is also present for an age of about 0.7 Gyr with a duration of about 0.2 Gyr. Burst 3 appears at 1.15 Gyr and is 0.1 Gyr wide. Unfortunately, it is difficult to recover burst 4 and 5 as the number of clusters older than 1.2 Gyr within $1 \mathrm{kpc}$ from the Sun is very low (3 objects). In fact, our results appear to suggest that the solar neighbourhood experienced quiescent star formation at 1.2-2 Gyr. From Fig. 12, the star formation rate history in the solar neighbourhood seems to be slightly different, with not a 


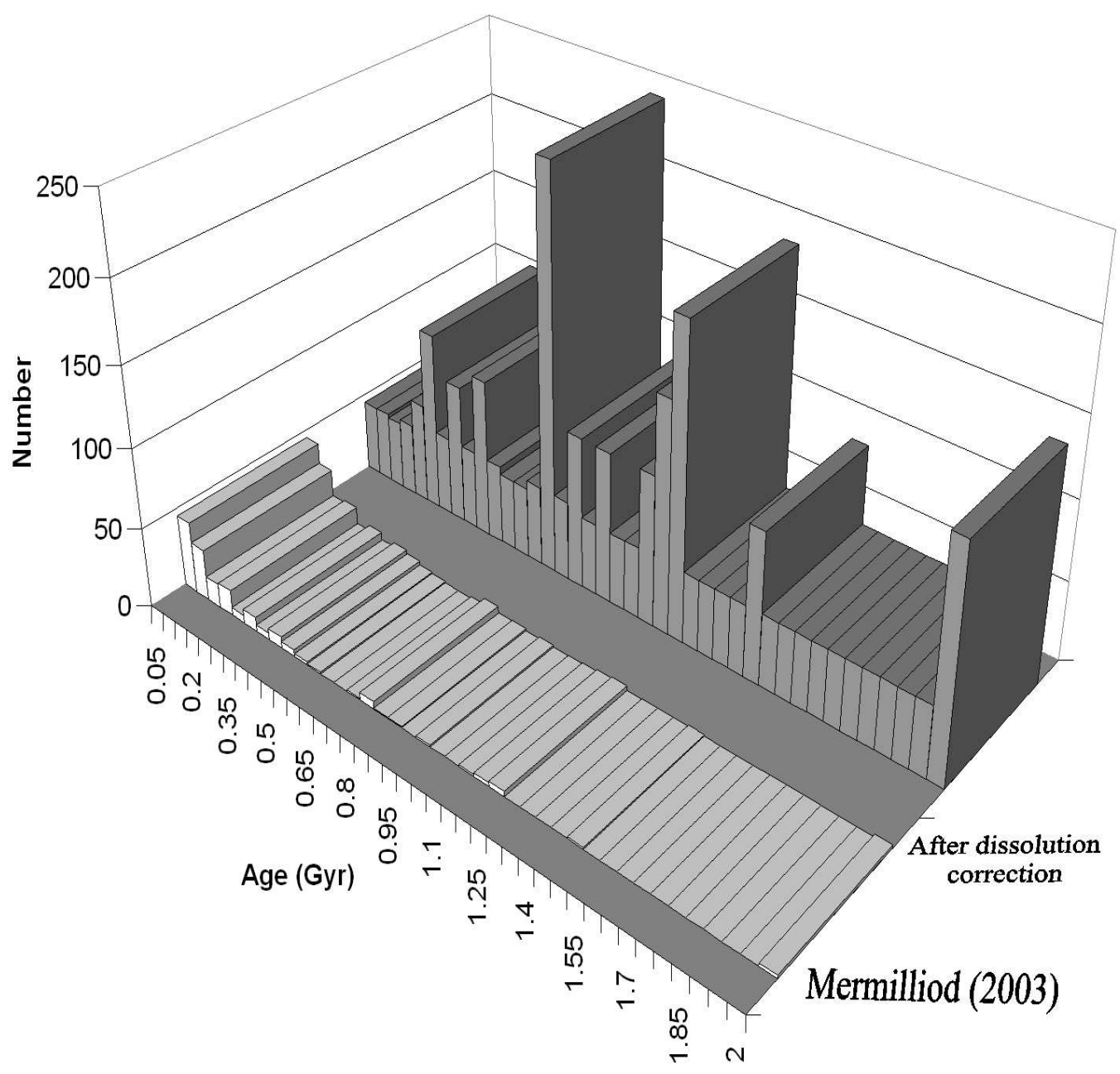

Fig. 13. Star formation history for the solar neighborhood (volume correction) after the dissolution corrections (see the text), compared to the age histogram used throughout this paper.

well defined period and narrower maxima. It is, however, easy to reconcile these apparent differences. The age distribution features interpreted as bursts 1,2 , and 3 are clearly identified in Fig. 12. Burst 4 and 5 may also exist but there are too few objects to confirm the hypothesis. On the other hand, uncertainties in the open cluster ages are smaller for closer clusters, therefore maxima are narrower and show better contrast against steady, quiescent star formation.

Fig. 13 shows the solar neighbourhood sample with evolutionary correction as described in the previous section. In this case the second burst is the strongest, followed by the third, the fifth, and the first. The fourth one is now the least important. 


\section{Comparison with other authors}

The problem of deducing the star formation history of our Galaxy has been primarily studied through chemical evolution models although other methods have also been used: Hertzsprung-Russell diagram inversion, stellar evolutionary tracks (Twarog, 1980; Meusinger, 1991; Bertelli \& Nasi, 2001), chromospheric activity as measured by Ca II and K emission (Barry, 1988; Soderblom et al., 1991; Rocha-Pinto et al. 2000a, b), stellar kinematics (Gomez et al., 1990; Marsakov et al. 1990; Chereul et al., 1998; Just, 2002, 2003), the mainsequence luminosity function (Scalo, 1987), the white dwarf luminosity function (Noh \& Scalo, 1990; Diaz-Pinto et al., 1994; Isern et al., 1999), combining the metallicity distribution and age-metallicity relation of G dwarfs (RochaPinto \& Maciel, 1997) and the distribution of coronal emission as measured by X-ray luminosities (Micela et al., 1993; Lachaume et al., 1999). Most of these studies have inferred an star formation history that is non-monotonic with time.

Although the majority of studies in this field use samples of stars in the solar neighbourhood with no stars more distant than about 100 pc being considered, it does not mean that the star formation history derived can only be applied to stars born in the solar neighbourhood. Nearby stars older than about 0.2 Gyr. come from birth sites which span a large range in Galactocentric distances. Wielen (1977) showed that the orbital diffusion coefficient deduced from the observed increase of velocity dispersion with age implies that such stars have suffered an rms azimuthal drift of about $2 \mathrm{kpc}$ for an age of 0.2 Gyr. Considerable, but smaller, drift should occur also in the radial direction. Wielen et al. (1996), on the basis of the Sun's metallicity and the radial metallicity gradient in the Galactic disk, estimated that the Sun has migrated outward by $1.9 \pm 0.9 \mathrm{kpc}$ in the past 4.5 Gyr. Sellwood and Binney (2002) have discussed the dynamics of radial migration due to transient spiral arms, and the relationship between radial migration and disk heating. They estimate that old stars formed in the solar neighbourhood should now be scattered nearly uniformly throughout an annulus of $8.5 \pm 4.0 \mathrm{kpc}$. In this sense the star formation history inferred for nearby stars is a measure of the global star formation history, at least at the Sun's Galactocentric radius. It is, however, not clear how the diffusion process affects entire star clusters not standalone stars and it is likely that star clusters experience little or no (azimuthal, radial) drift over the life-time. 
Table 1

Main features of the star formation history compared with other authors.

\begin{tabular}{llll}
\hline & This work & $\begin{array}{l}\text { Hernandez et al. } \\
(2000 \mathrm{a})\end{array}$ & $\begin{array}{l}\text { Rocha-Pinto et al. } \\
(2000 \mathrm{a})\end{array}$ \\
\hline Number of 'bursts' & 5 & 3 & 1 \\
Age of burst 1 & $0.4 \mathrm{Gyr}$ & $0.5 \mathrm{Gyr}$ & $0-1 \mathrm{Gyr}$ \\
Age of burst 2 & $0.8 \mathrm{Gyr}$ & $1.3 \mathrm{Gyr}$ & - \\
Age of burst 3 & $1.2 \mathrm{Gyr}$ & $1.9 \mathrm{Gyr}$ & - \\
Age of burst 4 & $1.5 \mathrm{Gyr}$ & - & - \\
Age of burst 5 & $1.9 \mathrm{Gyr}$ & - & - \\
\hline
\end{tabular}

\subsection{Hernandez et al. (2000a)}

Hernandez et al. (2000a) have derived the star formation history of the solar neighbourhood over the last 3 Gyr using data from the Hipparcos catalogue to construct color-magnitude diagrams. They treated these diagrams using advanced Bayesian analysis techniques (Hernandez et al., 1999; Hernandez et al., 2000) to deduce the star formation rate history of this region. They recovered the star formation history with an unprecedented time resolution of 0.05 Gyr. This high resolution makes it easy to compare our present results with those in their papers (Hernandez et al. 2000a, b, 2001). Their results indicate that the local star formation rate has an oscillatory component of period $\sim 0.5$ Gyr superimposed on a small level of constant star formation activity. Their inferred star formation history appears to be compatible with the observed distribution of stars. This cyclic behaviour is interpreted as the result of repeated encounters with the Galactic arm density pattern. In their work, they find that the last episode of enhanced star formation happened about $0.5 \mathrm{Gyr}$ ago. Another burst is found at about 1.3 Gyr and yet another one at 1.9 Gyr. Their Fig. 4 (Hernandez et al., 2000a) however suggests a double peak for the youngest burst and also for the one at 1.3 Gyr. In fact, their results appear to indicate one burst at about 0.4 Gyr (our burst 1) and another one at 0.7 Gyr (our burst 2). The 1.3 Gyr burst may be the convolution of burst 3 and 4 found in our analysis. Their third burst coincides in time with our fifth one. Therefore, our derivation of the recent star formation history both in the solar neighbourhood and the Galactic disk can be considered as fully consistent with the results found by Hernandez et al. (2000a) in spite of the fact that a completely different technique has been used in its derivation. 


\subsection{Other authors}

Rocha-Pinto et al. (2000a) have derived a star formation history from the chromospheric activity-age distribution of a large sample comprising 552 stars with Hipparcos parallaxes in the solar neighbourhood (stars more distant than 80 pc were omitted), and have found intermittency in the star formation rate over 14 Gyr. With a time resolution of 0.4 Gyr, their history of the star formation rate plotted in Fig. 2 (Rocha-Pinto et al., 2000a) and Table 1 (Rocha-Pinto et al., 2000b) indicates that the solar neighbourhood experienced a burst of star formation 0-1 Gyr ago (burst A). An epoch of low star formation took place 1-2 Gyr ago and a new, very strong burst was experienced 2-5 Gyr ago (burst B). An additional burst was found 7-9.5 Gyr ago (burst C). Although their time resolution makes a direct comparison impossible, their burst $\mathrm{A}$ is compatible with a convolution of our bursts 1 and 2, and possibly 3 . There are, however, strong discrepancies in the 1-2 Gyr range.

Noh and Scalo (1990) using the white dwarf luminosity function found peaks of star formation at 0.3 Gyr and $1.8 \mathrm{Gyr}$, that are compatible with our bursts 1 and 5. Barry (1988) using a technique similar to the one in Rocha-Pinto et al. (2000a), has also found three bursts using a volume-limited sample of 115 F-G stars, the most recent peaked in the last 0.5 Gyr (burst A). An epoch of low star formation took place 1-4 Gyr ago and a new burst was experienced 4-6 Gyr ago (burst B). The oldest burst was found 7-11 Gyr ago (burst C). His burst A appears to be a convolution of our bursts 1 and 2. Using the mainsequence luminosity function, Scalo (1987) found signatures of starbursts 5-6 and 0.3 Gyr ago. His 0.3 Gyr burst coincides with our burst 1. Twarog (1980) found that during the last 4 Gyr the star formation rate has remained more or less constant but there was a sharp increase from 4 to 8 Gyr ago. Although the time resolution in these studies is rather low $(0.2-1.0 \mathrm{Gyr})$, their results seem to indicate that bursts 1,2 , and 5 have also been identified by other authors.

\section{Age distribution maxima: enhanced star formation vs. accretion origin}

Starburst events are often seen in interacting galaxies, and it seems reasonable to infer a causal connection between galaxy interactions (mergers and close encounters) and starburst activity. It is now becoming increasingly clear that average galaxies engulf and devour smaller satellite galaxies as part of a more general process of hierarchical merging. Our own galaxy, the Milky Way, may have been incorporating dwarf galaxies into its disk since it was formed. The Galaxy is encircled by satellite galaxies that appear confined to one of two 
great streams across the sky (Lynden-Bell \& Lynden-Bell, 1995). The most well-known of these are the Magellanic Clouds and the associated HI Magellanic stream. All of these are expected to merge with the Milky Way in the distant future, largely due to the dynamical friction from the extended halo. The first strong evidence in favour of a hierarchical merging scenario for the Milky Way was presented by Ibata et al. $(1994,1995,1997)$ when they discovered the Sagittarius dwarf galaxy, a low mass dwarf spheroidal galaxy about $25 \mathrm{kpc}$ from the Sun in an advanced state of disruption that is being absorbed by the disk of the Milky Way. In fact, our results appear to indicate that the Sagittarius Dwarf Galaxy may have had some role in inducing enhanced star formation in the Milky Way disk. For about 10 years, the Sagittarius Dwarf Galaxy remained as the only strong evidence that the Milky Way is incorporating satellite galaxies, but another object has been added recently. The Canis Major Galaxy (Martin et al., 2004; Bellazzini et al., 2004) appears to be a close relative of the Sagittarius Dwarf Galaxy in terms of structural parameters. This new finding seems to confirm that part of the Galactic disk could be extragalactic in origin. On the other hand, recent numerical models of disk galaxy formation (Abadi et al., 2003a, b) indicate that disrupted galaxy satellites might have contributed a significant fraction of the old stars in the disk of the Milky Way. Navarro et al. (2004) have confirmed that the Arcturus Group, a dynamically-coherent group of 10-12 Gyr old, metal-deficient stars located in the solar neighbourhood around the star Arcturus, are very likely of extragalactic origin. These authors suggest that the Group is part of the tidal debris of a $10^{8} M_{\odot}$ disrupted satellite accreted by the Milky Way about 8 Gyr ago.

In this scenario, the peaks in the open cluster age distribution can be interpreted as signatures of merger events. This is however a non-straightforward interpretation as the variations found in the star formation rate appear to be periodic in nature, and mergers are expected to occur randomly in time. However, the actual sequence of events driving enhanced star formation episodes could be even more complex, with both tidal interactions and accretion events contributing to the global star formation history, as the unusual strength of burst 3 suggests.

For the main open cluster sample used in this work (Mermilliod, 2003), we find 97 clusters in the age range 0.2-0.6 Gyr and 80 objects in the age range 0.6-2.0 Gyr. There is a clear excess of clusters older than 0.6 Gyr. Strong star bursts are able to explain this feature but it could also be possible that some of these clusters may have been incorporated by the Galactic disk during accretion events in the recent past. In any case, the accretion scenario can only be important for coplanar interactions with the disk of the Milky Way and large accreted star clusters, otherwise the star clusters surviving the merger would end up either destroyed or in the Galactic halo. 


\subsection{The Canis Major galaxy role}

The chain of dynamical events that provoked the formation of the recently discovered Canis Major Dwarf Galaxy remnant (Martin et al., 2003; Bellazzini et al., 2003) has been proposed as the process that built up the Monoceros Ring (Newberg et al., 2002; Yanny et al., 2003; Ibata et al., 2003; Crane et al., 2003; Rocha-Pinto et al., 2003; Majewski et al., 2004). An unusually high spatial density of open clusters has been found in the field of the Canis Major galaxy. The list of open clusters includes, at least: NGC 2204, 2243, and 2477, Tombaugh 1 and 2, Berkeley 20 and 33, Melotte 66, and AM-2. Bellazzini et al. (2003) have also found that the position, distance, and stellar population of the old open clusters AM-2 and Tombaugh 2 strongly suggest that they are part of the Canis Major galaxy. Both are older than 4 Gyr and therefore they have not been included in our age distributions for clusters younger than 2 Gyr. Nevertheless, if the physical association between these open clusters and the merged dwarf galaxy is confirmed, it will be a clear example of intergalactic stellar pollution, with foreign star clusters being absorbed by the Galactic disk. If true, it may have a major impact on the determination of the star formation history of the Milky Way, with a fraction of the observed stars and open clusters being actually outsiders coming from other star formation histories.

\subsection{Searching for dwarf galaxy remnants}

In this paper we have shown that open clusters can be used as tracers to investigate the star formation history of the Milky Way disk. If a number of dwarf galaxies with their cohorts of star clusters have been captured and disrupted in the past, open clusters absorbed from these galaxies can be used to find the trail left by the parent galaxy. Searching for groups of coeval old open clusters with similar distances located in the same region of the sky can help to identify the bulk of the accreted satellite.

\section{Discussion and conclusions}

Does the Galaxy form stars continuously, or in bursts separated by epochs of relative quiescence? If star formation occurs in bursts, what processes mediate the bursts? These are just two of the many questions that we have tried to answer in this paper. We have applied a new method to carry out an objective reconstruction of the star formation history of the Milky Way disk over the last 2 Gyr. A sample composed of 581 open clusters with known ages and dis- 
tances was used in the derivation of this star formation history. Peaks in the age distribution diagram of the cluster sample were interpreted as signatures of star formation bursts. Our results indicate that the analyzed star formation rate presents two components: periodic episodes of enhanced star formation superimposed on a quiescent star formation level. A constant star formation history during the last 2 Gyr can therefore be discarded. A uniform rate of cluster formation and an exponentially declining dissolution rate would have produced a single population of clusters that would follow a straight line in a logarithmic plot with a slope given by the characteristic life-time of the population. Evidence for at least five epochs of enhanced star formation during the time interval studied has been found. The recent star formation history derived in this work is consistent with star formation rate histories deduced using a range of other techniques. Interpreting the age distribution diagram suggests that quiescent (non-enhanced) star formation has proceeded in discrete, highly-correlated regions of activity producing clusters which dissipate their parent molecular cloud on a time-scale of a few Myr $(<10 \mathrm{Myr})$. The majority of open clusters formed during the non-enhanced star formation periods are destroyed and their members become field stars on a time-scale < 20 Myr. However, there appears to be a long-lasting component as well, since some of the clusters are able to survive for over 0.2 Gyr. During bursts of star formation, the huge number of clusters formed make it possible to produce larger than average objects that could survive for several Gyr. A number of simulations were done in order to estimate the impact of the age errors on the features found in the age distribution diagram. Even for age errors larger than $30 \%$ some of these features (the younger ones) are still clearly identifiable. On the other hand, we have examined the possibility that the Galactic bursts are coeval with features in the star formation history of the Magellanic Clouds and the Sagittarius Dwarf Galaxy, as well as with close encounters between them and the Milky Way. Although the degree of uncertainty is large, there are several coincidences that suggest tidal interactions can play some role as inductive forces of bursts of star formation in the Milky Way disk. Distant galactic encounters may trigger significant star formation but quiescent star formation in the disk of the Milky Way appears to be characterized by a large number of small, short lived open clusters that contribute a small fraction of the total number of stars formed ( $<150$ per cluster) with a small number of large, relatively long lived clusters that may contribute most of the stars in the disk.

There is, however, a cyclic behaviour in the burst sequence that may be better explained by the density wave hypothesis (Lin \& Shu, 1964) for the presence of spiral arms in late-type galaxies. A model like the one outlined in Hernandez et al. (2000a) and developed in Martos et al. (2004) can explain easily the 0.4 Gyr periodicity that we detect. Heating by spiral structure can explain the main features of the age-velocity dispersion relation and other parameters of the velocity distribution in the solar neighbourhood, like why the stars in a 
single velocity-space moving group have a wide range of ages (De Simone et al., 2004). For a pattern speed $\Omega_{p}=20 \mathrm{~km} \mathrm{~s}^{-1} \mathrm{kpc}^{-1}$ (e.g. Martos et al., 2004) and a Galactocentric distance $R_{\odot}=8.5 \mathrm{kpc}$ it implies an orbital period of about 1 Gyr for the Sun. If the enhanced star formation episodes are, in fact, due to the interactions with the spiral arms it means that our Galaxy has two arms. This has been recently suggested by Martos et al. (2004) using a completely different approach. On the other hand, studies on the nature of star formation triggering in large disks indicate that a number of mechanisms may operate concurrently: gravitational instabilities, shocks between colliding clouds of gas, enhanced pressure of the interstellar medium, strong stellar winds, supernova shocks (star formation triggered by nearby bursts of star formation), density waves, shear forces produced by differential rotation, and interactions or mergers with other galaxies. Star formation triggered by previous star formation events is a self-propagating process which may continue over a much longer period of time, hence it is possible that overall a relatively large fraction of star formation is triggered.

On the other hand, if the star formation history inferred from the age distribution of open clusters is consistent with that deduced for individual stars then one can consider this as an argument in favour of most of the observed stars being formed in some kind of star clusters. Since only a subsample of the young clusters are likely to survive, an obvious question is whether most of the field stars in a galaxy are originally formed in clusters. Even in these young star forming regions many of the field stars are from clusters that have already been dissolved, hence the true percentage of stars that were originally in clusters is even higher, and might conceivably be $\approx 100 \%$. It is tempting to suggest that perhaps the majority of stars are formed in groups and clusters, and that the field stars are simply the remnants of the fainter, less dense clusters which have dissolved. In fact, infrared observations show that most star formation occurs in embedded star clusters within Giant Molecular Clouds. These clusters have masses in the range $50-10^{3} M_{\odot}$ (Lada \& Lada, 2003). Nevertheless, it is still possible to consider the existence of two channels in the star formation process: the dispersed mode and the clustered mode, by modifying slightly our concepts of dispersed and clustered. The dispersed mode would be represented by the numerous short lived $(\tau<30 \mathrm{Myr})$ small star clusters that are part of the quiescent star formation rate. These are different from classical open clusters and may be called Fast Living Clusters or flusters in opposition to the fully fledged standard open clusters as the Hyades or Pleiades. The clustered mode would be represented by the relatively long lived open clusters. In our analysis of the last 0.2 Gyr of star formation, the number of clusters in the age range 30-100 remains relatively constant as well as the fraction in the age range 100-200. This feature suggests a real gap in the mass distribution of disk star clusters: very few clusters are formed in the mass range 20-30 $M_{\odot}(N=50-75)$. To explain the very good matching between the star formation history inferred from individual stars and that from open star 
clusters we have to assume that although most of open clusters $(>90 \%)$ are poor, and short lived $(N \leq 200)$ most of the stars $(>90 \%)$ are formed in larger clusters $(N>200)$. This conclusion, however, appears not to be supported by current observations.

The third largest galaxy in the Local Group, Triangulum galaxy (M33, NGC 598) may be undergoing star formation events similar to the ones described in this paper. M33 is a spiral galaxy about half the size of the Milky Way. Chandar et al. (1999) have found 44 young clusters in M33 with ages $\leq 100$ Myr and masses in the range $6 \times 10^{2}$ to $2 \times 10^{4} M_{\odot}$. Currently, M33 appears to be forming many young compact clusters but less massive than an older cluster population formed in a previous starburst with characteristic mass $\sim 10^{5} M_{\odot}$.

Although our conclusions are uncertain as they are based on a relatively small (36\%) subsample of a larger but still incomplete sample composed of almost 2000 open clusters, they are similar to the ones previously found by a number of other independent studies. This confirms the validity of our approach to infer star formation histories (recent and old), not just for the Milky Way disk but for any other galaxy. On the other hand, if the conclusions obtained in this paper are correct, the Galactic disk will start another episode of enhanced star formation within the next very few Myr if it is not already happening right now.

\section{Acknowledgements}

We thank the referee whose comments greatly improved this paper. We are very grateful to S.J. Aarseth for providing his computer code and for his comments on the manuscript. We thank the Department of Astrophysics of Universidad Complutense of Madrid for providing excellent computing facilities at the Centro de Proceso de Datos in Moncloa. The authors thank S. van den Bergh, L. O. Lodén, J.-C. Mermilliod, G. Carraro, and I. Platais for very helpful and interesting discussions. We also thank M. Martos, X. Hernández and associated group for sharing with us their results on the Galactic spiral pattern and its rotation speed in advance of publication. In preparation of this paper we made use of the Open Cluster Database, operated at the Institut d'Astronomie de l'Université de Lausanne, Switzerland, the New Catalogue of Optically Visible Open Clusters and Candidates, operated at the Universidade de São Paulo, Brazil, the NASA Astrophysics Data System and the ASTRO-PH e-print server. 


\section{References}

[1] Aarseth, S.J., 1985, in: Multiple Time Scales, eds. J.U. Brackbill \& B.I. Cohen, Academic Press, New York, p. 377.

[2] Aarseth, S.J., 1994, in: Galactic Dynamics and N-body Simulations, eds. G. Contopoulos, N.K. Spirou, \& L. Vlahos, Springer-Verlag, Berlin, p. 277.

[3] Aarseth, S.J., 1999, PASP, 111, 1333.

[4] Aarseth, S.J., 2003, Gravitational N-Body Simulation: Tools and Algorithms (Cambridge University Press, Cambridge).

[5] Aarseth, S.J. \& Zare, K., 1974, CeM, 10, 185.

[6] Abadi, M.G., Navarro, J.F., Steinmetz, M., \& Eke, V.R., 2003a, ApJ, 591, 499.

[7] Abadi, M.G., Navarro, J.F., Steinmetz, M., \& Eke, V.R., 2003b, ApJ, 597, 21.

[8] Ahmad, A. \& Cohen L., 1973, J. Comput. Phys., 12, 389.

[9] Ann, H.B., Lee, S.H., Sung, H., Lee, M.G., Kim, S.-L., Chun, M.-Y., Jeon, Y.-B., Park, B.-G., \& Yuk, I.-S., 2002, AJ, 123, 905.

[10] Barry, D.C., 1988, ApJ, 334, 436.

[11] Battinelli, P., Brandimarti, A., \& Capuzzo-Dolcetta, R., 1994, A\&AS, 104, 379.

[12] Baume, G., Villanova, S., \& Carraro, G., 2003, A\&A, 407, 527.

[13] Baumgardt, H., 2001, MNRAS, 325, 1323.

[14] Baumgardt, H. \& Makino, J., 2003, MNRAS, 340, 227.

[15] Bellazzini, M., Ibata, R.A., Monaco, L., Martin, N.F., Irwin, M.J., \& Lewis, G.F., 2004, MNRAS, (in press).

[16] van den Bergh, S., 2000, PASP, 112, 932.

[17] Bertelli, G. \& Nasi, E., 2001, AJ, 121, 1013.

[18] Bik, A., Lamers, H.J.G.L.M., Bastian, N., Panagia, N., \& Romaniello, M., 2003, A\&A, 397, 473.

[19] Binney, J. \& Tremaine, S., 1987 Galactic Dynamics (Princeton University Press, Princeton).

[20] Bressan, A., Fagotto, F., Bertelli, G., \& Chiosi, C., 1993, A\&AS, 100, 647.

[21] Brown, A.G.A., 2002, in: Modes of Star Formation and the Origin of Field Populations, eds. E.K. Grebel \& W. Brandner (ASP Conference Series), p. 150.

[22] Bruntt, H., Frandsen, S., Kjeldsen, H., \& Andersen, M.I., 1999, A\&AS, 140, 135 . 
[23] Carraro, G., Hassan, S.M., Ortolani, S., \& Vallenari, A., 2001, A\&A, 372, 879.

[24] Carraro, G., Barbon, R., \& Boschetti, C.S., 2002, MNRAS, 336, 259.

[25] Carraro, G. \& Munari, U., 2004, MNRAS, 347, 625.

[26] Chabrier, G., 2003, PASP, 115, 763.

[27] Chandar, R., Bianchi, L., Ford, H.C., Salasnich, B., 1999, PASP, 111, 794.

[28] Chereul, E., Crezé, M., \& Bienaymé, O., 1998, A\&A, 340, 384.

[29] Clarke, C.J., Bonnell, I.A., \& Hillenbrand, L.A., 2000, in: Protostars and Planets IV, eds. V. Mannings, A.P. Boss, \& S.S. Russell (University of Arizona Press), p. 151.

[30] Comerón, F., et al., 2002, A\&A, 389, 874.

[31] Crane, J.D., Majewski, S.R., Rocha-Pinto, H.J., Frinchaboy, P.M., Skrutskie, M.F., \& Law, D.R., 2003, ApJ, 594, L119.

[32] Dias, W.S., Alessi, B.S., Moitinho, A., \& Lépine, J.R.D., 2002, A\&A, 389, 871.

[33] Dias, W.S., Alessi, B.S., Moitinho, A., \& Lépine, J.R.D., 2003a, in: Galactic and Stellar Dynamics, eds. C.M. Boily, P. Patsis, S. Portegies Zwart, R. Spurzem, \& C. Theis, EAS Publications Series, p. 195.

[34] Dias, W.S., Alessi, B.S., Moitinho, A., \& Lépine, J.R.D., 2003b, New catalogue of optically visible open clusters and candidates - version 1.4, http://www.astro.iag.usp.br/ wilton

[35] Díaz-Pinto, A., Garcia-Berro, E., Hernanz, M., Isern, J., \& Mochkovitch, R., 1994, A\&A, 282, 86.

[36] Eggleton, P.P., Fitchett, M.J., \& Tout, C.A., 1989, ApJ, 347, 998.

[37] Elmegreen, B.G., Efremov, Y., Pudritz, R.E., \& Zinnecker, H., 2000, in: Protostars and Planets IV, eds. V. Mannings, A.P. Boss, \& S.S. Russell (University of Arizona Press), p. 179.

[38] ESA, 1997, The Hipparcos catalogue, ESA SP-1200.

[39] Freeman, K. \& Bland-Hawthorn, J., 2002, ARA\&A, 40, 487

[40] de la Fuente Marcos, R., 1993, unpublished dissertation, Universidad Complutense de Madrid.

[41] de la Fuente Marcos, R., 1995, A\&A, 301, 407.

[42] de la Fuente Marcos, R., 1996a, A\&A, 308, 141.

[43] de la Fuente Marcos, R., 1996b, A\&A, 314, 453.

[44] de la Fuente Marcos, R., 1997a, A\&A, 322, 764. 
[45] de la Fuente Marcos, R., 1997b, Ph.D. Thesis, Universidad Complutense de Madrid.

[46] de la Fuente Marcos, R., 1998, A\&A, 333, L27.

[47] de la Fuente Marcos, R. \& de la Fuente Marcos, C., 2000, Ap\&SS, 271, 127.

[48] de la Fuente Marcos, R. \& de la Fuente Marcos, C., 2002, Ap\&SS, 371, 1097.

[49] de la Fuente Marcos, R. \& de la Fuente Marcos, C., 2004, (in preparation).

[50] Gardiner, L.T., Sawa, T., \& Fujimoto, M., 1994, MNRAS, 266, 567.

[51] Gizis, J.E., Reid, I.N., \& Hawley, S.L., 2002, AJ, 123, 3356.

[52] Gomez, A.E., Dehaye, J., Grenier, S., Jaschek, C., Arenou, F., \& Jaschek, M., 1990, A\&A, 236, 95.

[53] Gray, D.F., 1965, AJ, 70, 362.

[54] Grocholski, A.J. \& Sarajedini, A., 2002, AJ, 123, 1603.

[55] Hanson, M.M., 2003, ApJ, 597, 957.

[56] Harris, J. \& Zaritsky, D., 2004, AJ, (in press)

[57] Hernandez, X., Gilmore, G., \& Valls-Gabaud, D., 2000, MNRAS, 317, 831.

[58] Hernandez, X., Valls-Gabaud, D., \& Gilmore, G., 1999, MNRAS, 304, 705.

[59] Hernandez, X., Valls-Gabaud, D., \& Gilmore, G., 2000a, MNRAS, 316, 605.

[60] Hernandez, X., Valls-Gabaud, D., \& Gilmore, G., 2000b, in: The Evolution of the Milky Way: stars versus clusters, eds. F. Matteucci \& F. Giovannelli (Kluwer Academic Publishers), p. 517.

[61] Hernandez, X., Valls-Gabaud, D., \& Gilmore, G., 2001, in: Galaxy Disks and Disk Galaxies, eds. J.G. Funes \& E.M. Corsini (ASP Conference Series), p. 17.

[62] Ibata, R.A., Gilmore, G., \& Irwin, M.J., 1994, Nature, 370, 194.

[63] Ibata, R.A., Gilmore, G., \& Irwin, M.J., 1995, MNRAS, 277, 781.

[64] Ibata, R.A., Wyse, R.F.G., Gilmore, G., Irwin, M.J., \& Suntzeff, N.B., 1997, AJ, 113, 634 .

[65] Ibata, R.A., Irwin, M.J., Lewis, G.F., Ferguson, A.M.N., \& Tanvir, N., 2003, MNRAS, 340, L21.

[66] Isern, J., Hernanz, M., Garcia-Berro, E., \& Mochkovitch, E., 1999, in: 11th European Workshop on White Dwarfs, eds. J.E. Solheim \& E.G. Meistas (ASP Conference Series), p. 408.

[67] Janes, K.A., Tilley, C., \& Lyngå, G., 1988, AJ, 95, 771.

[68] Johnston, K.V., 1998, ApJ, 495, 297. 
[69] Johnston, K.V., Majewski, S.R., Siegel, M.H., Reid, I.N., \& Kunkel, W.E., 1999, AJ, 118, 1719.

[70] Just, A., 2002, in: Disks of Galaxies: Kinematics, Dynamics and Perturbations, eds. E. Athanassoula, A. Bosma, \& R. Mujica (ASP Conference Series), p. 117.

[71] Just, A., 2003, Ap\&SS, 284, 727.

[72] Knödlseder, J., 2000, A\&A, 360, 539.

[73] Knödlseder et al., 2002, A\&A, 390, 945.

[74] Kroupa, P., 1995a, MNRAS, 277, 1491.

[75] Kroupa, P., 1995b, MNRAS, 277, 1507.

[76] Kroupa, P., 1995c, MNRAS, 277, 1491.

[77] Kroupa, P., 2002, Science, 295, 82.

[78] Kroupa, P. \& Tout, C.A., 1992, MNRAS, 259, 223.

[79] Kroupa, P. \& Boily, C.M., 2002, MNRAS, 336, 1188.

[80] Kroupa, P., Tout, C.A., \& Gilmore, G., 1990, MNRAS, 244, 76.

[81] Kroupa, P., Tout, C.A., \& Gilmore, G., 1991, MNRAS, 251, 293.

[82] Kroupa, P., Tout, C.A., \& Gilmore, G., 1993, MNRAS, 262, 545.

[83] Kroupa, P., Aarseth, S.J., \& Hurley, J., 2001, MNRAS, 321, 699.

[84] Kustaanheimo, P. \& Stiefel, E.L., 1965, J. Reine Angew. Math., 218, 204.

[85] Lachaume, R., Dominik, C., Lanz, T., \& Habing, H.J., 1999, A\&A, 348, 897.

[86] Lada, C.J. \& Lada, E.A., 1991, in: The Formation and Evolution of Star Clusters, ed. K. Janes, ASP Conference Series, p. 3.

[87] Lada, C.J. \& Lada, E.A., 2003, ARA\&A, 41, 57.

[88] Larsen, S.S., \& Richtler, T., 2000, A\&A, 354, 836.

[89] Lata, S., Pandey, A.K., Sagar, R., \& Mohan, V., 2002, A\&A, 388, 158.

[90] Lin, C.C. \& Shu, F.H., 1964, ApJ, 140, 646.

[91] Lin, D.N.C., Jones, B.F., \& Klemola, A.R., 1995, ApJ, 439, 652.

[92] Lynden-Bell, D. \& Lynden-Bell, R.M., 1995, MNRAS, 275, 429.

[93] Lyngå, G., 1985, in: The Milky Way Galaxy, IAU Symp. 106, eds. H. van Woerden, R.J. Allen, \& W.B. Burton (Dordrecht: Reidel), p. 243.

[94] Lyngå, G., 1987, Catalogue of Open Cluster Data, 5th ed. (Cent. Données Stellaire, Strabourg). (available at http://cdsweb.u-strasbg.fr) 
[95] Majewski, S.R., 1993, ARA\&A, 31, 575.

[96] Majewski, S.R., Skrutskie, M.F., Weinberg, M.D., Ostheimer, J.C., 2004, ApJL, (in press).

[97] Marsakov, V.A., Shevelev, Y.G., \& Suchkov, A.A., 1990, Ap\&SS, 172, 51.

[98] Martin, N.F., Ibata, R.A., Bellazzini, M., Irwin, M.J., Lewis, G.F., \& Dehnen, W., 2004, MNRAS, 348, 12.

[99] Martos, M., Hernández, X., Yáñez, M., Moreno, E., \& Pichardo, B., 2004, MNRAS, (submitted).

[100] Mermilliod, J.-C., 1988, Inform. Bull. CDS, 35, 77.

[101] Mermilliod, J.-C., 1992a, in: Astronomy from Large Databases II, eds. A. Heck \& F. Murtagh, ESO Conf. and Work. Proc., 43, 373.

[102] Mermilliod, J.-C., 1992b, Inform. Bull. CDS, 40, 115.

[103] Mermilliod, J.-C., 1993, in: Databases for Galactic Structure, eds. A.G.D. Philip, B. Hauck, \& A.R. Upgren (L. Davis Press: Schenectady), p. 27.

[104] Mermilliod, J.-C., 1995, in: Information and on-line data in Astronomy, eds. D. Egret \& M.A. Albrecht, (Dordrecht: Kluwer), p. 127.

[105] Mermilliod, J.-C., 1996, in: The Origins, Evolutions, and Destinies of Binary Stars in Clusters, eds. E.F. Milone \& J.-C. Mermilliod (ASP Conference Series), p. 475.

[106] Mermilliod, J.-C., 2003, Open Cluster Database, http://www.unige.ch/webda.

[107] Meusinger, H., 1991, Ap\&SS, 182, 19.

[108] Meyer, M.R., Adams, F.C., Hillenbrand, L.A., Carpenter, J.M., \& Larson, R.B., 2000, in: Protostars and Planets IV, eds. V. Mannings, A.P. Boss, \& S.S. Russell (University of Arizona Press), p. 121.

[109] Micela, G., Sciortino, S., \& Favata, F., 1993, ApJ, 412, 618.

[110] Mikkola, S., 1985, MNRAS, 215, 171.

[111] Mikkola, S. \& Aarseth, S.J., 1993, CeMDA, 57, 439.

[112] Miller, G.E. \& Scalo, J.M., 1979, ApJS, 41, 513.

[113] Murai, T. \& Fujimoto, M., 1980, PASJ, 32, 581.

[114] Navarro, J.F., Helmi, A., \& Freeman, K.C., 2004, ApJL, (in press).

[115] Newberg, N.J., et al., 2002, ApJ, 569, 245.

[116] Noh, H.-R. \& Scalo, J., 1990, ApJ, 352, 605. 
[117] Pandey, A.K., Bhatt, B.C., Mahra, H.S., \& Sagar, R., 1989, MNRAS, 236, 263.

[118] Piskunov, A.E., Belikov, A.N., Kharchenko, N.V., Sagar, R., \& Subramaniam, A., 2004, MNRAS, (in press).

[119] Popper, D.M., 1980, ARA\&A, 18, 115.

[120] Prisinzano, L., Micela, G., Sciortino, S., \& Favata, F., 2004, A\&A, (in press).

[121] Reddish, V.C., 1978, Stellar Formation (Pergamon Press).

[122] Roberts, M.S., 1957, PASP, 69, 59.

[123] Rocha-Pinto, H.J. \& Maciel, W.J., 1997 MNRAS, 289, 882.

[124] Rocha-Pinto, H.J., Scalo, J., Maciel, W.J., \& Flynn, C., 2000a, ApJ, 531, L115.

[125] Rocha-Pinto, H.J., Scalo, J., Maciel, W.J., \& Flynn, C., 2000b, A\&A, 358, 869 .

[126] Rocha-Pinto, H.J., Majewski, S.R., Skrutskie, M.F., \& Crane, J.D., 2003, ApJ, 594,115 .

[127] Sagar, R., Joshi, U.C., \& Sinvhal, S.D., 1983, Bull. Astron. Soc. India, 11, 44.

[128] Salaris, M., Weiss, A., \& Percival, S.M., 2004, A\&A, 414, 163.

[129] Salpeter, E.E., 1955, ApJ, 121, 161.

[130] Scalo, J.M., 1986, Fundam. Cosmic Phys., 11, 1.

[131] Scalo, J.M., 1987, in: Starbursts and Galaxy Evolution, eds. T.X. Thuan, T. Montmerle, \& Tran Thanh Van (Editions Frontières: Gif sur Yvette), p. 445.

[132] Schaller, G., Schaerer, D., Meynet, G., \& Maeder, A., 1992, A\&AS, 96, 269.

[133] Sellwood, J.A. \& Binney, J.J., 2002, MNRAS, 336, 785.

[134] De Simone, R.S., Wu, X., \& Tremaine, S., 2004, MNRAS, (in press).

[135] Soderblom, D.R., Duncan, D.K., \& Johnson, D.R., 1991, ApJ, 375, 722.

[136] Spassova, N.M. \& Beav, P.V., 1985, Ap\&SS, 112, 111.

[137] Taff, L.G., 1974, AJ, 79, 11.

[138] Terlevich, E., 1983, Ph.D. Thesis, University of Cambridge.

[139] Terlevich, E., 1985, in: Dynamics of Star Clusters, eds. J. Goodman \& P. Hut, D. Reidel Publ., p. 471.

[140] Terlevich, E., 1987, MNRAS, 224, 193.

[141] Twarog, B.A., 1980, ApJ, 242, 242.

[142] Vázquez, R.A. \& Feinstein, A., 1989, Rev. Mexicana Astron. Astrof., 17, 3. 
[143] Whitmore, B.C., Zhang, Q., Leitherer, C., Fall, S.M., Schweizer, F., \& Miller, B.W., 1999, AJ, 118, 1551.

[144] Wielen, R., 1977, A\&A, 60, 263.

[145] Wielen, R., Fuchs, B., \& Dettbarn, C., 1996, A\&A, 314, 438.

[146] Yanny, B., et al., 2003, ApJ, 588, 824.

[147] Zaritsky, D. \& Harris, J., 2004, AJ, (in press).

[148] Zombeck, M.V., 1990, Handbook of Astronomy and Astrophysics, 2nd Ed., Cambridge University Press, Cambridge. 